\title{
Administration of antioxidant peptide SS-31 attenuates transverse aortic constriction-induced pulmonary arterial hypertension in mice
}

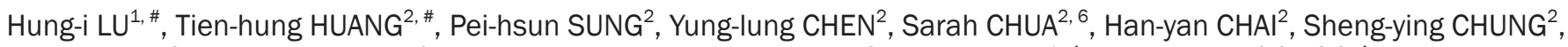
Chu-feng LIU ${ }^{3}$, Cheuk-kwan SUN ${ }^{4}$, Hsueh-wen CHANG ${ }^{5}$, Yen-yi ZHEN², Fan-yen LEE ${ }^{1, *}$, Hon-kan YIP², 6, 7,8,9, *

\begin{abstract}
${ }^{1}$ Division of Thoracic and Cardiovascular Surgery, Department of Surgery; ${ }^{2}$ Division of Cardiology, Department of Internal Medicine; ${ }^{3}$ Department of Emergency Medicine, Kaohsiung Chang Gung Memorial Hospital and Chang Gung University College of Medicine, Kaohsiung, Taiwan, China; ${ }^{4}$ Department of Emergency Medicine, E-Da Hospital, I-Shou University School of Medicine for International Students, Kaohsiung, Taiwan, China; ${ }^{5}$ Department of Biological Sciences, National Sun Yat-Sen University, Kaohsiung, Taiwan, China; ${ }^{6}$ Institute for Translational Research in Biomedicine; ${ }^{7}$ Center for Shock Wave Medicine and Tissue Engineering, Kaohsiung Chang Gung Memorial Hospital and Chang Gung University College of Medicine, Kaohsiung, Taiwan, China; ${ }^{8}$ Department of Medical Research, China Medical University Hospital, China Medical University, Taichung, Taiwan, China; ${ }^{9}$ Department of Nursing, Asia University, Taichung, 41354, Taiwan, China
\end{abstract}

\begin{abstract}
Aim: Antioxidant peptide SS-31 is a class of cell-permeable small peptides, which selectively resides on the inner mito $\neg$ chondrial membrane and possesses intrinsic mitochondrial protective capacities. In this study we investigated the therapeutic effects of antioxidant peptide SS-31 on transverse aortic constriction (TAC)-induced pulmonary arterial hypertension (PAH) in a murine model. Methods: Adult male mice were divided into 3 groups: sham-operated mice, TAC mice, and TAC+SS-31 mice that underwent TAC surgery and received SS-31 ( $2 \mathrm{mg} / \mathrm{d}$, ip) for $60 \mathrm{~d}$. The right ventricular systolic blood pressure (RVSBP) was measured on d 60 prior to sacrificing the mice; then their right heart and lung tissues were collected for histological and biochemical examinations. Lung injury scores were defined by the increased crowded area and decreased number of alveolar sacs.

Results: TAC mice showed significantly higher RVSBP compared with sham-operated mice, the elevation was substantially suppressed in TAC+SS-31 mice. The same pattern of changes was found in pulmonary levels of oxidative stress proteins (NOX-1/NOX-2/oxidized proteins), cytosolic cytochrome $c$, biomarkers related to inflammation (MMP-9/TNF- $\alpha /$ iNOS), calcium overload index (TRPC1, 2, 4, 6 ), apoptosis (mitochondrial BAX, cleaved caspase 3/PARP), fibrosis (Smad3/TGF- $\beta$ ), hypoxic (HIF-1 $\alpha$ ), DNA damage ( $\gamma$-H2AX) and endothelial function (eNOS/ET-1R), as well as in lung injury score, number of muscularized vessels in lungs, number of TRPC1 ${ }^{+}$and $\mathrm{HIF}-1 \alpha^{+}$cells in pulmonary artery, and number of $\mathrm{y}-\mathrm{H} 2 \mathrm{AX} \mathrm{X}^{+}$and $\mathrm{Ki}-67^{+}$cells in lung parenchyma. An opposite pattern of changes was observed in pulmonary anti-fibrotic markers (Smad1/5, BMP-2), number of small vessels, and number of alveolar sacs. In contrast, the levels of antioxidant proteins (HO-1/NQO-1/GR/GPx) in lung parenchyma were progressively and significantly increased from shamoperated mice, TAC mice to TAC+SS-31 mice.

Conclusion: Antioxidant peptide SS-31 administration effectively attenuates TAC-induced PAH in mice.
\end{abstract}

Keywords: antioxidant peptide SS-31; mitochondria-targeted; pulmonary arterial hypertension; transverse aortic constriction; ROS; inflammation; apoptosis

Acta Pharmacologica Sinica (2016) 37: 589-603; doi: 10.1038/aps.2015.162; published online 11 Apr 2016

\section{Introduction}

It is well recognized that the late stage of both systolic and

\footnotetext{
\# These authors contributed equally to this work.

* To whom correspondence should be addressed.

E-mail han.gung@msa.hinet.net

Received 2015-09-24 Accepted 2015-12-25
}

diastolic left ventricular failure frequently causes pulmonary edema, pulmonary hypertension, and right-sided heart failure regardless of the etiology ${ }^{[1-5]}$. Therefore, advanced left-sided and right-sided heart failure (HF) are actually two sides of the same coin in pathophysiology. Abundant data have shown that patients with advanced HF are frequently re-admitted due to recurrent $\mathrm{HF}^{[1-3]}$, which results in major health care 
expenses worldwide ${ }^{[6,7]}$. In addition, although the etiology and mechanisms of HF have been extensively investigated and various therapeutic strategies have been developed ${ }^{[8-10]}$, the morbidity and mortality of patients with advanced HF are still extremely high ${ }^{[1-3,6-8]}$. Accordingly, the development of novel therapeutic options is of the utmost importance for scientists conducting basic research and especially for physicians who are the first-line managers of these patients.

Reactive oxygen species (ROS) generation and mitochondrial dysfunction caused by oxidative injury, which are two important contributors to apoptosis or necrosis ${ }^{[1-13]}$, are implicated in the pathophysiology of $\mathrm{HF}^{[11,14,15]}$. Moreover, mitochondrial oxidative damage promotes mitochondrial cytochrome $c$ release ${ }^{[16]}$ and cessation of ATP production. These could explain the markedly impaired diastolic or systolic heart function of both right and left ventricles in patients with advanced HF.

Because mitochondria are known to be both the source and target of $\operatorname{ROS}^{[13]}$, a number of previous studies have explored the possibility of mitochondria-targeted therapeutic approaches for the treatment of different diseases ${ }^{[12,13,16-19]}$. Antioxidant peptide SS-31, which is a novel class of cell-permeable small peptides, selectively resides on the inner mitochondrial membrane and possesses intrinsic mitochondrial protective capacities ${ }^{[17,18,20]}$. Moreover, previous experimental studies have shown that SS-31 can scavenge ROS, reduce mitochondrial ROS production, and inhibit mitochondrial permeability transition ${ }^{[16-18,20]}$. Additionally, SS-31 has been shown to be potent in preventing the cell apoptosis and necrosis induced by oxidative stress or inhibition of the mitochondrial electron transport chain, especially in animal models of acute ischemia-reperfusion injury ${ }^{[16-18,20]}$. Surprisingly, while the therapeutic role of peptide SS-31 in acute heart ischemiareperfusion injury and hypertensive cardiomyopathy has been reported $^{[17,19]}$, the potential of this drug in treating left-sided heart failure-induced pulmonary arterial hypertension (PAH) has not been explored. Accordingly, this study aims to investigate the therapeutic potential of peptide SS-31, a mitochondrial targeted antioxidant, for the treatment of TAC-induced PAH by utilizing animal models.

\section{Materials and methods Ethics}

All animal experimental procedures were approved by the Institute of Animal Care and Use Committee at Chang Gung Memorial Hospital - Kaohsiung Medical Center (Affidavit of Approval of Animal Use Protocol No. 2015032403) and were performed in accordance with the Guide for the Care and Use of Laboratory Animals (NIH publication № 85-23, National Academy Press, Washington, DC, USA, revised 1996).

\section{Animal grouping}

Pathogen-free adult male C57BL/6 (B6) mice $(n=36)$, weighing 22-25 gm, (Charles River Technology, BioLASCO Taiwan Co, Ltd, Taiwan), were randomized and equally categorized into sham control (SC) (receiving thoracotomy only), transverse aortic constriction (TAC only), and TAC+SS-31 (2 mg intraperitoneal injection/day for total 60 days) groups, respectively. The dosage of $2 \mathrm{mg}$ intraperitoneal injection/day for the animals was based on a previous report ${ }^{[18]}$ with minimal modification.

Four lung specimens of these animals from each group were collected for determination of the number of alveolar sacs and crowded score, while the remaining eight lung specimens were used for other individual studies. Additionally, four RV specimens of these animals were collected for determination of fibrotic area and collagen deposition area, and the remainders were used for RT-PCR and determination of brain natriuretic peptide (BNP) protein expression.

\section{Procedure of transverse aortic constriction}

The mice were anesthetized by inhalation of $2.0 \%$ isoflurane. Atropine $0.05 \mathrm{mg} / \mathrm{kg}$ was administered subcutaneously. A core body temperature of approximately $37^{\circ} \mathrm{C}$ was maintained during surgery by continuous monitoring with a rectal thermometer and automatic heating blanket. The mice were endotracheally intubated with positive-pressure ventilation using $100 \%$ oxygen with a tidal volume of $250 \mu \mathrm{L}$ at a rate of 120 breaths per minute using a Small Animal Ventilator (SAR830/A, CWE, Inc, USA). After opening the chest wall via an upper sternotomy, the aorta was carefully identified. A 25\# needle was placed on the aorta, on which a 7-0 prolene ligation was placed between the right and left common carotid arteries. The needle was then carefully removed, and the aortic constriction was created. After the procedure, the wound was closed, and the animal was allowed to recover from anesthesia in a portable animal intensive care unit (ThermoCare $^{\circledR}$ ) for $24 \mathrm{~h}$.

\section{Hemodynamic studies and specimen preparation}

The procedure and protocol were based on our previous report ${ }^{[21]}$. In detail, on day 60 after TAC induction, the mice were anesthetized by inhalation of $2.0 \%$ isoflurane. Each animal was endotracheally intubated with positive-pressure ventilation using $100 \%$ oxygen with a tidal volume of $250 \mu \mathrm{L}$ at a rate of 120 breaths per minute using a Small Animal Ventilator (SAR-830/A, CWE, Inc, USA). The heart was exposed by left thoracotomy. A sterile 20-gauge, soft plastic needle was inserted into the right ventricle and femoral artery of each mouse to measure the right ventricular systolic blood pressure (RVSBP). The pressure signals were first transmitted to pressure transducers (UFI, model 1050, CA, USA) and then exported to a bridge amplifier (ML866 PowerLab 4/30 Data Acquisition Systems, ADInstruments Pty Ltd, Castle Hill, NSW, Australia) where the signals were amplified and digitized. The data were recorded and later analyzed using Labchart software (ADInstruments). After hemodynamic measurements were taken, the mice were euthanized, and the right hearts and lungs were harvested.

Pilot study for assessing TAC-induced pulmonary arterial hypertension

To elucidate whether the PAH animal model was successfully 
created by the TAC procedure, an additional ten mice were equally utilized as SC and TAC only. On day 60, the RVSBP, an index of pulmonary arterial systolic blood pressure (PABP), was measured in these animals. The results showed that the RVSBP and the total heart weight were remarkably increased in the TAC group compared with the SC group (Figure 1). Additionally, the gross anatomy showed that the heart size was notably larger in the TAC group than in the SC group (Figure 1). These findings showed that the TAC-induced PAH was successfully created.

\section{Western blot analysis of lung and heart tissue}

The procedure and protocol for Western blot analysis were based on our recent reports ${ }^{[21-23]}$. Briefly, equal amounts (50
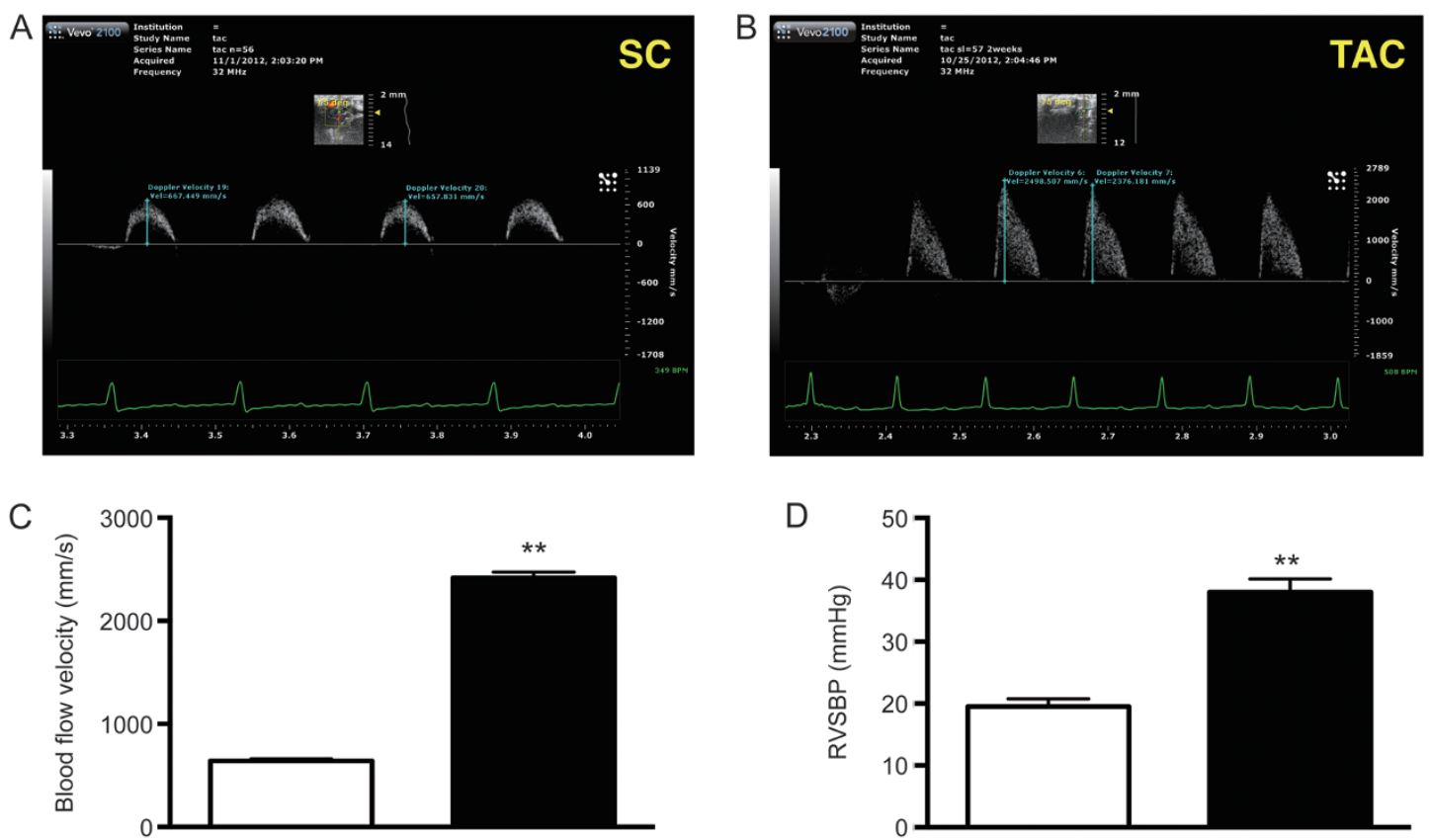

E
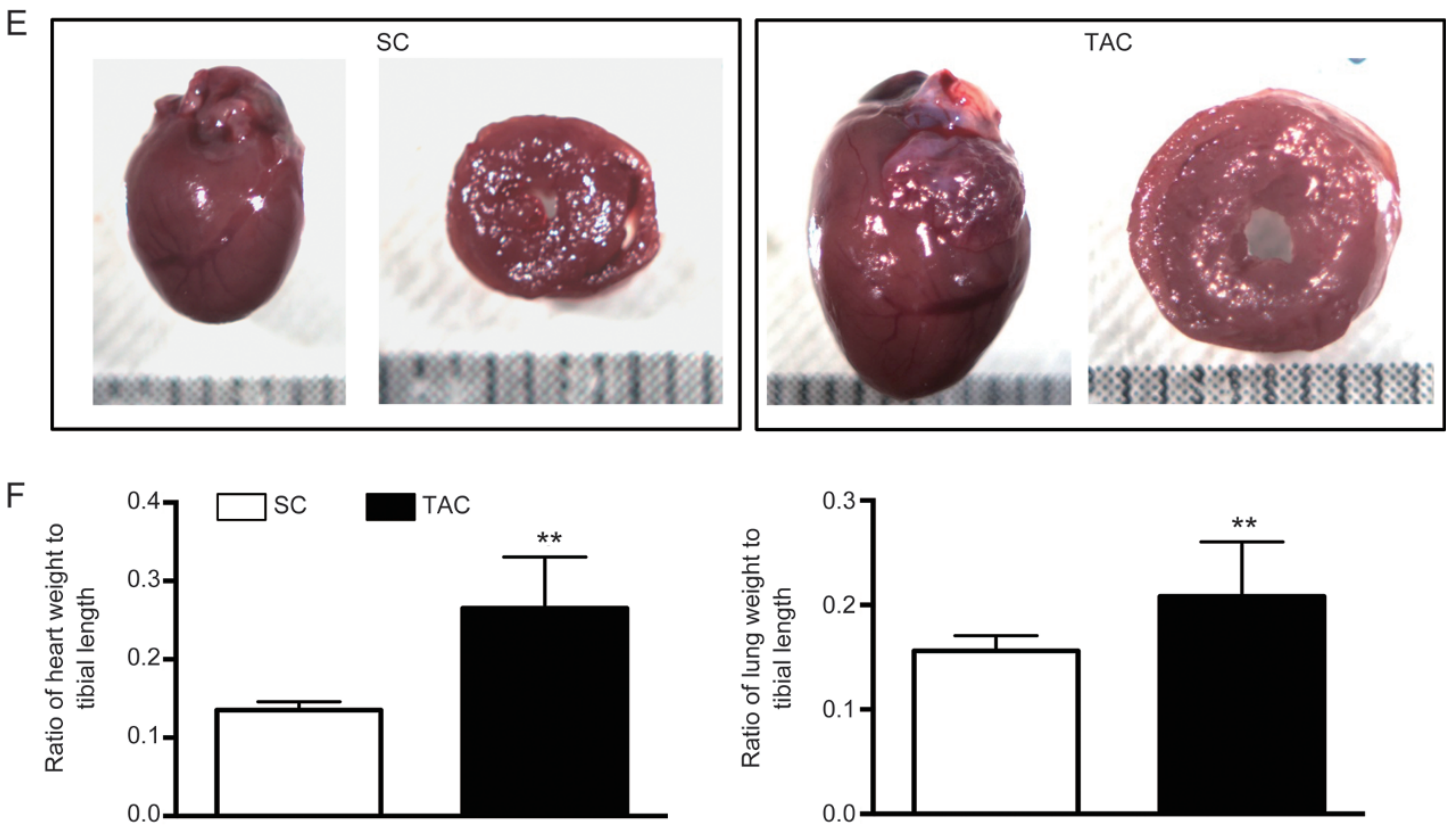

Figure 1. Results of the pilot study. (A \& B) The Doppler results of blood flow velocity of the ascending aorta 2 weeks after the transverse aortic constriction (TAC) procedure. (C) Analytical results of blood flow velocity at the ascending aorta. (D) Right ventricular systolic blood pressure (RVSBP) at day 60 after TAC procedure. (E) The gross anatomical finding of the appearance of the whole heart and a cross section of the heart at the papillary level. Notably larger size of the whole heart and left ventricular hypertrophy (ie, from cross section) were observed in TAC animals compared with sham control (SC) animals. (F) The ratio of heart weight to tibial length. (G) The ratio of lung weight to tibial length. Mean \pm SEM. $n=6$ for each group. ${ }^{* *} P<0.01$ vs TAC group. 
$\mu \mathrm{g})$ of protein extracts were loaded and separated by SDSPAGE using acrylamide gradients. After electrophoresis, the separated proteins were transferred electrophoretically to a polyvinylidene difluoride (PVDF) membrane (Amersham Pharmacia Biotech Inc, USA). Nonspecific sites were blocked by incubation of the membrane in blocking buffer [5\% nonfat dry milk in T-TBS (TBS containing $0.05 \%$ Tween 20)] overnight. The membranes were incubated with the indicated primary antibodies [Bax (1: 1000, Abcam), cleaved poly (ADPribose) polymerase (PARP) (1:1000, Cell Signaling), Bcl-2 (1:200, Abcam), phosphorylated (p)-Smad3 (1:1000, Cell Signaling), p-Smad1/5 (1:1000, Cell Signaling), bone morphogenetic protein (BMP) 2 (1:5000, Abcam), transforming growth factor (TGF)- $\beta$ (1:500, Abcam), matrix metalloproteinase (MMP)-9 (1:3000, Abcam), tumor necrosis factor (TNF)-a (1:1000, Cell Signaling), nuclear factor (NF)-кB (1:600, Abcam), endothelial nitric oxide synthase (eNOS) (1:1000, Abcam), endothelin 1 receptor (ET-1R) (1:1000, Abcam), brain natriuretic peptide (BNP) (1:600, Abcam), transient receptor potential cation channel (TRPC) 1 (1:1000, Abcam) TRPC4 (1:600, Abcam), TRPC6 (1:1000, Abcam), hypoxic inducible factor (HIF)-1a (1:750, Abcam), heme oxygenase (HO)-1 (1:500, Millipore), NAD(P) $\mathrm{H}$ quinone oxidoreductase (NQO) 1 (1:1000, Abcam), glutathione reductase (GR) (1:1000, Abcam), glutathione peroxidase (GPx) (1:1000, Abcam), and actin (1:10000, Chemicon)] for 1 $\mathrm{h}$ at room temperature. Horseradish peroxidase-conjugated anti-rabbit immunoglobulin IgG (1:2000, Cell Signaling) was used as a secondary antibody for one-hour of incubation at room temperature. The washing procedure was repeated eight times within one hour. Immunoreactive bands were visualized by enhanced chemiluminescence (ECL; Amersham Biosciences) and exposed to Biomax L film (Kodak). For quantification, ECL signals were digitized using Labwork software (UVP).

\section{Oxidative stress reaction in lung parenchyma}

The procedure and protocol for assessing the expression of proteins associated with oxidative stress have previously been described in detail in our previous reports ${ }^{[21-23]}$. The Oxyblot Oxidized Protein Detection Kit was purchased from Chemicon (S7150). DNPH derivatization was carried out on $6 \mu \mathrm{g}$ of protein for 15 min according to the manufacturer's instructions. One-dimensional electrophoresis was carried out on $12 \%$ SDS/polyacrylamide gel after DNPH derivatization. Proteins were transferred to nitrocellulose membranes, which were then incubated in the primary antibody solution (anti-DNP 1: 150) for $2 \mathrm{~h}$, followed by incubation in the secondary antibody solution (1:300) for $1 \mathrm{~h}$ at room temperature. The washing procedure was repeated eight times within 40 minutes. Immunoreactive bands were visualized by enhanced chemiluminescence (ECL; Amersham Biosciences) and exposure to Biomax $\mathrm{L}$ film (Kodak). For quantification, ECL signals were digitized using Labwork software (UVP). For OxyBlot protein analysis, a standard control was loaded on each gel.

\section{Immunofluorescence (IF) staining}

IF staining was performed for the examination of $\gamma-\mathrm{H}_{2} \mathrm{AX}^{+}$, $\mathrm{TRPC}^{+} \mathrm{Ki}^{-67^{+}} \mathrm{HIF}-1 \mathrm{a}^{+}$cells and troponin-I in LV myocardium using the respective primary antibodies based on our recent study ${ }^{[21-23]}$. Irrelevant antibodies were used as controls in the current study.

\section{Vessel density and arterial muscularization in lung parenchyma} The procedure and protocol for assessing the number of small vessels were described in detail in our previous reports ${ }^{[22,24]}$. IHC staining of small blood vessels was performed with a-SMA (1:400) as a primary antibody at room temperature for $1 \mathrm{~h}$, followed by washing with PBS three times. Ten minutes after the addition of anti-mouse HRP-conjugated secondary antibody, the tissue sections were washed with PBS three times. Then, 3,3'-diaminobenzidine (DAB) $(0.7 \mathrm{mg} /$ tablet $)$ (Sigma) was added, followed by washing with PBS three times after one minute. Finally, hematoxylin was added as a counter-stain for nuclei, followed by washing twice with PBS after one minute. Three heart sections were analyzed from each mouse. For quantification, three randomly selected high power fields $(\mathrm{HPFs})(\times 200)$ were analyzed in each section. The mean number per HPF for each animal was then determined by summation of all of the numbers divided by 9 .

Muscularization of the arterial medial layer (ie, an index of vascular remodeling) in lung parenchyma was defined as a mean thickness of the vessel wall greater than $50 \%$ of the luminal diameter in a vessel of diameter $>50 \mu \mathrm{m}$. Measurement of arteriolar diameter and wall thickness was achieved using ImageJ software (NIH, Maryland, USA).

\section{Real-time quantitative PCR analysis of RV myocardium}

Real-time polymerase chain reaction (PCR) was conducted using LightCycler TaqMan Master (Roche) in a single capillary tube according to the manufacturer's guidelines regarding the concentrations of individual components. Forward and reverse primers were each designed in a different exon of the target gene sequence, eliminating the possibility of amplifying genomic DNA. A positive result was determined by identifying the threshold cycle value at which reporter dye emission appeared above background. If fluorescence signal was not detected within 33 cycles, the sample was considered negative.

\section{Assessment of the distribution of alveolar Sacs and crowded score in lung parenchyma by immunohistochemical (IHC) staining}

The procedure and protocol for determination of the number of alveolar sacs were based on our previous report ${ }^{[22]}$. In detail, three lung sections from each mouse were analyzed and three randomly selected HPFs $(\times 100)$ were examined in each section. The number of alveolar sacs was recorded for each animal. The mean number per HPF for each animal was then determined by summation of all of the numbers divided by 9 . The percentage of crowded area (defined as septal thickness associated with partial or complete collapse of alveoli) in lung 
parenchyma was determined using $\mathrm{H}$ \& $\mathrm{E}$ staining in a blind fashion and scored as follows: $0=$ no detectable crowded area; $1=<15 \%$ of crowded area; $2=15 \%-25 \%$ of crowded area; $3=$ $25 \%-50 \%$ of crowded area; $4=50 \%-75 \%$ of crowded area; $5=$ $>75 \%-100 \%$ of crowded area/per high-power field $(\times 100)$.

\section{Histological quantification of myocardial fibrosis and collagen} deposition in RV

The procedure and protocol were described in detail in our previous report ${ }^{[25]}$. In detail, Masson's trichrome staining was used for identifying the fibrosis of RV myocardium. Three $4-\mu \mathrm{m}$-thick serial sections from the same levels of the RV myocardium from each animal were prepared using a Cryostat (Leica CM3050S). The integrated area $\left(\mu \mathrm{m}^{2}\right)$ of infarct area and fibrosis on each section were calculated using the Image Tool 3 (IT3) image analysis software (University of Texas, Health Science Center, San Antonio, UTHSCSA; Image Tool for Windows, Version 3.0, USA). Three randomly selected highpower fields (HPFs) $(\times 100)$ were analyzed in each section. After determining the number of pixels in each infarct and the fibrotic area per HPF, the numbers of pixels obtained from three HPFs were summated. The procedure was repeated in two other sections for each animal. The mean pixel number per HPF for each animal was then determined by summating all pixel numbers and dividing by 9 . The mean integrated area $\left(\mu \mathrm{m}^{2}\right)$ of fibrosis in RV myocardium per HPF was obtained using a conversion factor of $19.24\left(1 \mu \mathrm{m}^{2}\right.$ represented 19.24 pixels).

To analyze the extent of collagen synthesis and deposition, cardiac paraffin sections $(6 \mu \mathrm{m})$ were stained with picrosirius red ( $1 \%$ Sirius red in saturated picric acid solution) for one hour at room temperature using standard methods. The sections were then washed twice with $0.5 \%$ acetic acid. The water was physically removed from the slides by vigorous shaking. After dehydration in $100 \%$ ethanol three times, the sections were cleaned with xylene and mounted in a resinous medium. High power fields $(\times 100)$ of each section were used to identify the Sirius red-positive area on each section. Analyses of collagen deposition area in RV myocardium were identical to the description for the calculations of the infarct and fibrotic areas.

\section{Statistical analysis}

Quantitative data are expressed as the mean \pm SD. Statistical analysis was adequately performed by ANOVA followed by Bonferroni multiple-comparison post hoc test. SAS statistical software for Windows version 8.2 (SAS institute, Cary, NC) was utilized. A probability value $<0.05$ was considered statistically significant.

\section{Results}

Pilot study results of anatomical, pathological, and hemodynamic findings on day 60 after the TAC procedure

To determine whether TAC was able to induce PAH through cardiac hypertrophy (ie, an outcome of passive PAH), right ventricular hemodynamic measurements (ie, an indicator of pulmonary artery blood pressure), blood flow velocity of the ascending aorta, total heart weight and gross anatomic features of the heart were analyzed. On day 60 after the TAC procedure, the peak systolic velocity of blood flow in the aorta (ie, a reflection of the pressure gradient between the left ventricle and the aorta) was notably increased in the TAC group compared with the sham-operated controls (SC) (Figure 1). Additionally, RVSBP was substantially elevated in the TAC group compared with RVSBP in the SC group (Figure 1). Furthermore, the size and total heart weight were significantly increased in the TAC group compared with those in the SC group (Figure 1). These findings suggest the successful creation of an experimental model of hypertensive cardiomyopathy with PAH.

Gene and protein expression levels as well as hemodynamic and histopathological findings of the right ventricle on day 60 after the TAC procedure

On day 60 after TAC, the changes in gene expression levels of NOX-1 and NOX-2 (ie, two indicators of ROS generation) as well as TNF-a, MMP-9, and inducible nitric oxide synthase (iNOS) (ie, three biomarkers of inflammation) were significantly higher in the TAC group than in the SC group (Figure 2). Cardiac hypertrophy is characterized by a switch from $\alpha$ - to $\beta$-myosin heavy chain (MHC) mRNA expression (ie, reactivation of the fetal gene program). In the current study, mRNA expression of $\beta$-MHC in RV was significantly higher in the TAC group than in the SC group, whereas a-MHC in RV showed a reverse pattern between the two groups (Figure 2). Moreover, the protein expression levels of BNP (ie, an indicator of pressure or volume overload) showed an identical pattern of ROS between the two groups (Figure 2). Again, these parameters were significantly reversed in TAC animals with SS-31 treatment.

Masson's trichrome staining showed that the RV fibrotic area was significantly increased in TAC group compared with that of the SC group (Figure 3). Consistently, Sirius red staining revealed that the RV collagen deposition area showed an identical pattern of fibrosis in these two groups (Figure 3). These two parameters were significantly reversed in TAC animals with SS-31 treatment.

The protein expression of cytosolic cytochrome $c$ of the right ventricle, an indicator of mitochondrial damage, was significantly higher in the TAC group than in the SC and TAC+ SS-31 groups and was significantly higher in the TAC+SS-31 group than in the SC group. On the other hand, the total protein expression of mitochondrial cytochrome $c$, an indicator of mitochondrial integrity, showed an opposite pattern of cytosolic cytochrome c among the three groups.

Histopathological changes in lung parenchyma on day 60 after the TAC procedure

IHC staining demonstrated a significantly reduced number of small vessels (ie, <15 $\mu \mathrm{m}$ in diameter) and number of alveolar sacs in animals with TAC compared to those without (Figure 4). On the other hand, the differences in crowded score and the number of muscularized pulmonary arterioles between the 
A
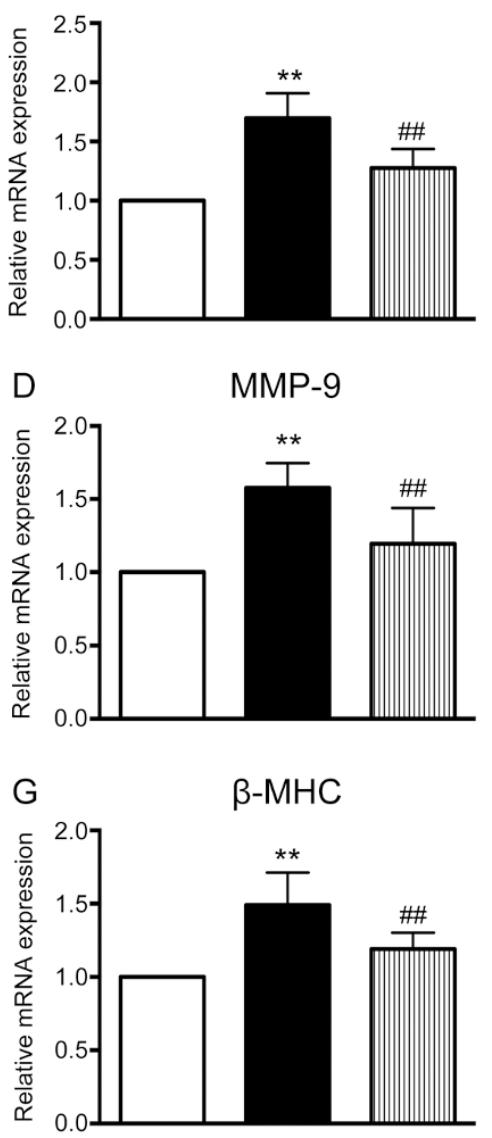

B
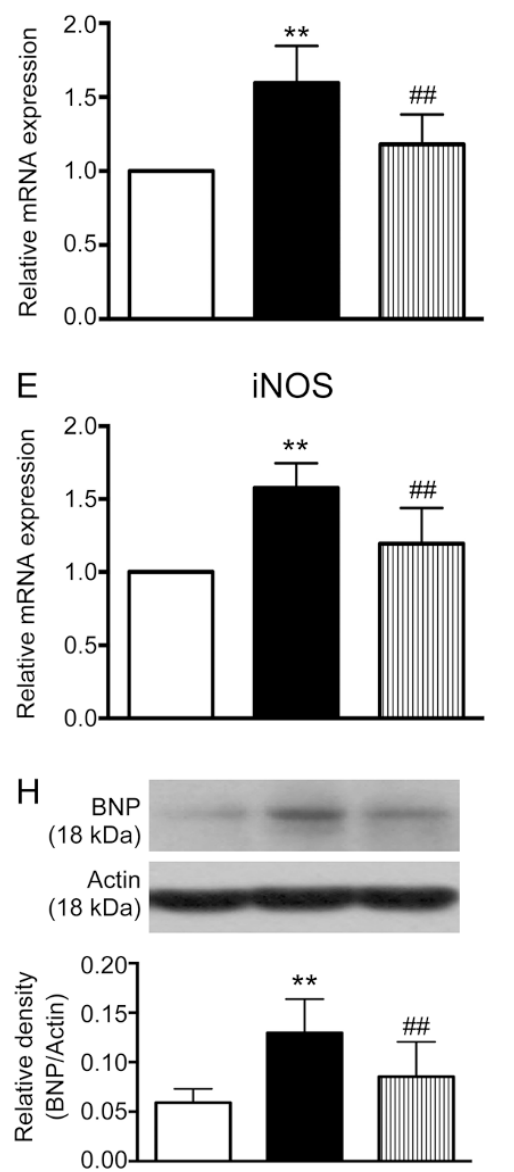

C

TNF- $\alpha$

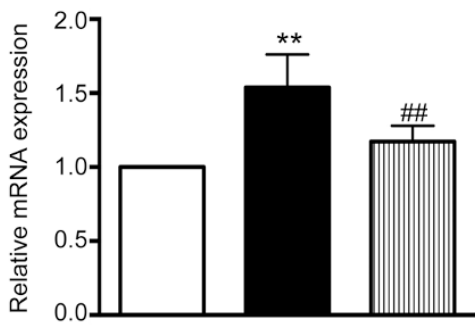

F

a-MHC

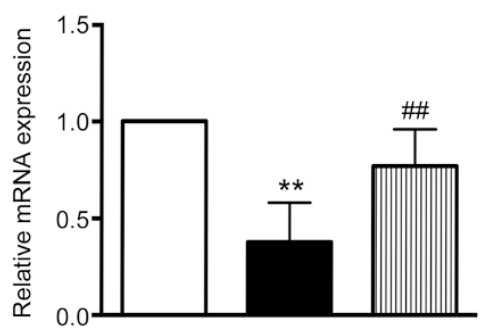

I

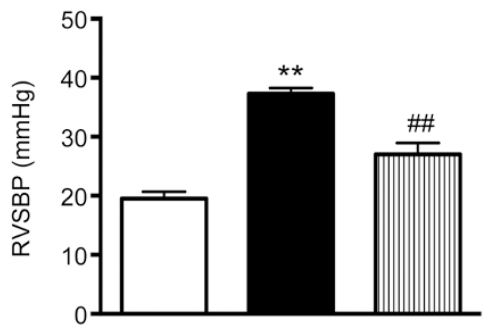

Figure 2. Gene and protein expression levels and RVSBP on day 60 after the TAC procedure. (A) mRNA expression of NOX-1. (B) mRNA expression of NOX-2. (C) mRNA expression of tumor necrosis factor (TNF)- $\alpha$. (D) mRNA expression of matrix metalloproteinase (MMP)-9. (E) mRNA expression of inducible nitric oxide synthase (iNOS). (F) mRNA expression of $\alpha$-myosin heavy chain (MHC). (G) mRNA expression of $\beta$-MHC. (H) Protein expression of brain natriuretic peptide (BNP). (I) Analytical results of RVSBP. All statistical analyses were performed using one-way ANOVA followed by Bonferroni multiple comparison post hoc test. $\mathrm{SC}=$ sham control. TAC=transverse aortic constriction. Mean $\pm \mathrm{SEM} . n=6$ for each group. ${ }^{* *} P<0.01$ vs $\mathrm{SC}$ group. ${ }^{\# \#} P<0.01$ vs TAC group.

TAC and SC animals showed an opposite pattern compared to that of the number of alveolar sacs (Figure 4). Additionally, the pattern of immunofluorescent expression of TRPC1 ${ }^{+}$ cells (ie, an index of hypoxia-inducible biomarker related to smooth muscle proliferation) and $\mathrm{Ki}-67^{+}$cells [for quantifying SMC proliferation in the medial layer of large pulmonary artery (PA)] in pulmonary arteriolar smooth muscle (Figure 5) and HIF-1a ${ }^{+}$(ie, another hypoxia-inducible biomarker) and

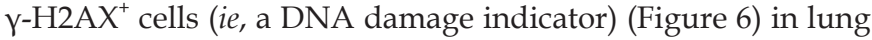
parenchyma were identical to that of the number of alveolar sacs in the two groups of animals. Importantly, the expression levels of all of the biomarkers were reversed in TAC animals after SS-31 treatment.

Changes in protein expression levels in lung parenchyma on day 60 after the TAC procedure

The protein expression levels of NOX-1 and NOX-2, oxidative stress, ET-1R, Y-H2AX (Figure 7), cytosolic cytochrome $c$, HIF-1a, TRPC1, TRPC4, and TRPC6 (Figure 8) were signifi- cantly higher in TAC animals than those in SC animals and in TAC animals with SS-31 treatment. The levels of these proteins were significantly higher in TAC animals that received SS-31 treatment compared to SC animals, whereas mitochondrial cytochrome $c$ showed a reverse pattern among the three groups of animals (Figure 8).

The protein expression levels of inflammatory (ie, TNFa, NF-кB, and MMP-9), apoptotic (ie, mitochondrial Bax, cleaved caspase 3, and PARP) and fibrotic (ie, Smad3 and TGF- $\beta$ ) biomarkers were significantly higher in TAC animals than in SC animals and were significantly lower in TAC animals after SS-31 treatment (Figure 9). On the other hand, protein expression levels of anti-fibrotic (Smad1/5, BMP-2), anti-inflammatory (Bcl-2) and endothelial function (eNOS) biomarkers showed an opposite pattern compared to that of the inflammatory biomarkers among the three groups (Figure 10). Furthermore, the protein expression levels of antioxidants (HO-1, NQO 1, GR, and GPx) were significantly increased in TAC animals and were further significantly increased in TAC 

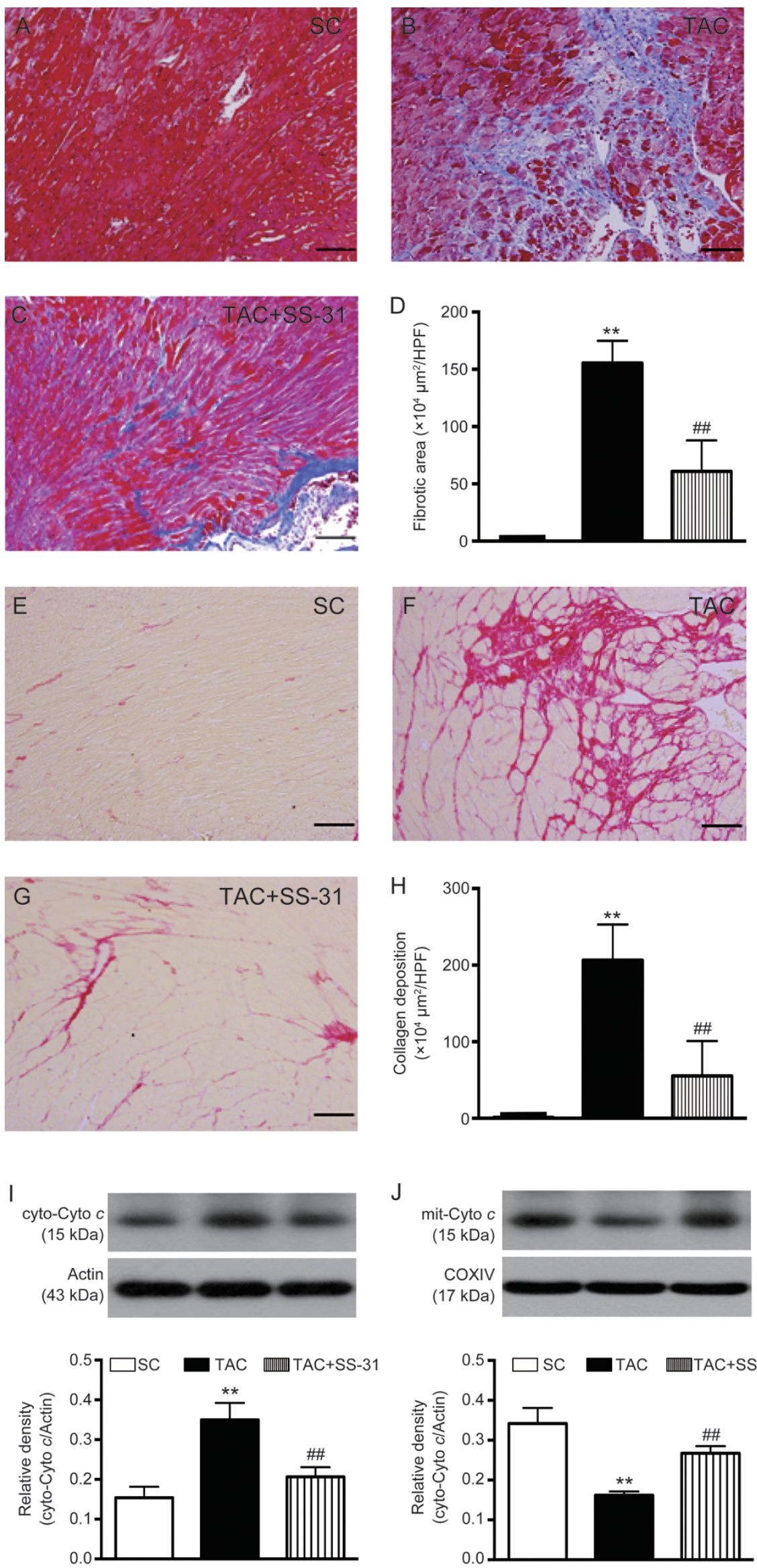
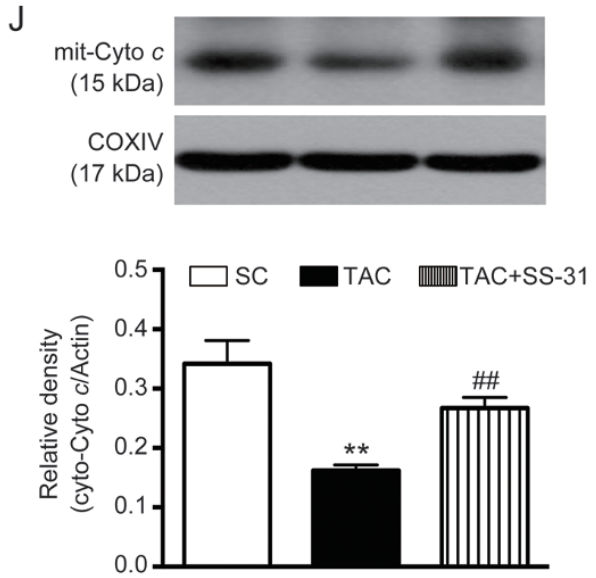

Figure 3. Histopathological changes and protein expression levels of cytochrome $\mathrm{c}$ of the right ventricle on day 60 after the TAC procedure. (A-C) Microscopic observation of Masson's trichrome staining for the identification of fibrotic area $(\times 100)$. (D) Analytical result of fibrotic area. The scale bars in the right lower corner represent $100 \mu \mathrm{m}$. $(E-G)$ Microscopic observation of Sirius red staining for identifying the collagen deposition area. (H) Analytical result of fibrotic area. The scale bars in the right lower corner represent $100 \mu \mathrm{m}$. (I) Protein expression of cytosolic cytochrome c (cyt-Cyto c). (J) Protein expression of mitochondrial cytochrome $c$ (mit-Cyto $c$ ). All statistical analyses were performed using oneway ANOVA followed by Bonferroni multiple comparison post hoc test. $\mathrm{SC}=$ sham control. $\mathrm{TAC}=$ transverse aortic constriction. $\mathrm{HPF}=$ highpower field. Mean \pm SEM. $n=6$ for each group. ${ }^{* *} P<0.01$ vs SC group. ${ }^{\# \#} P<0.01$ vs TAC group. 

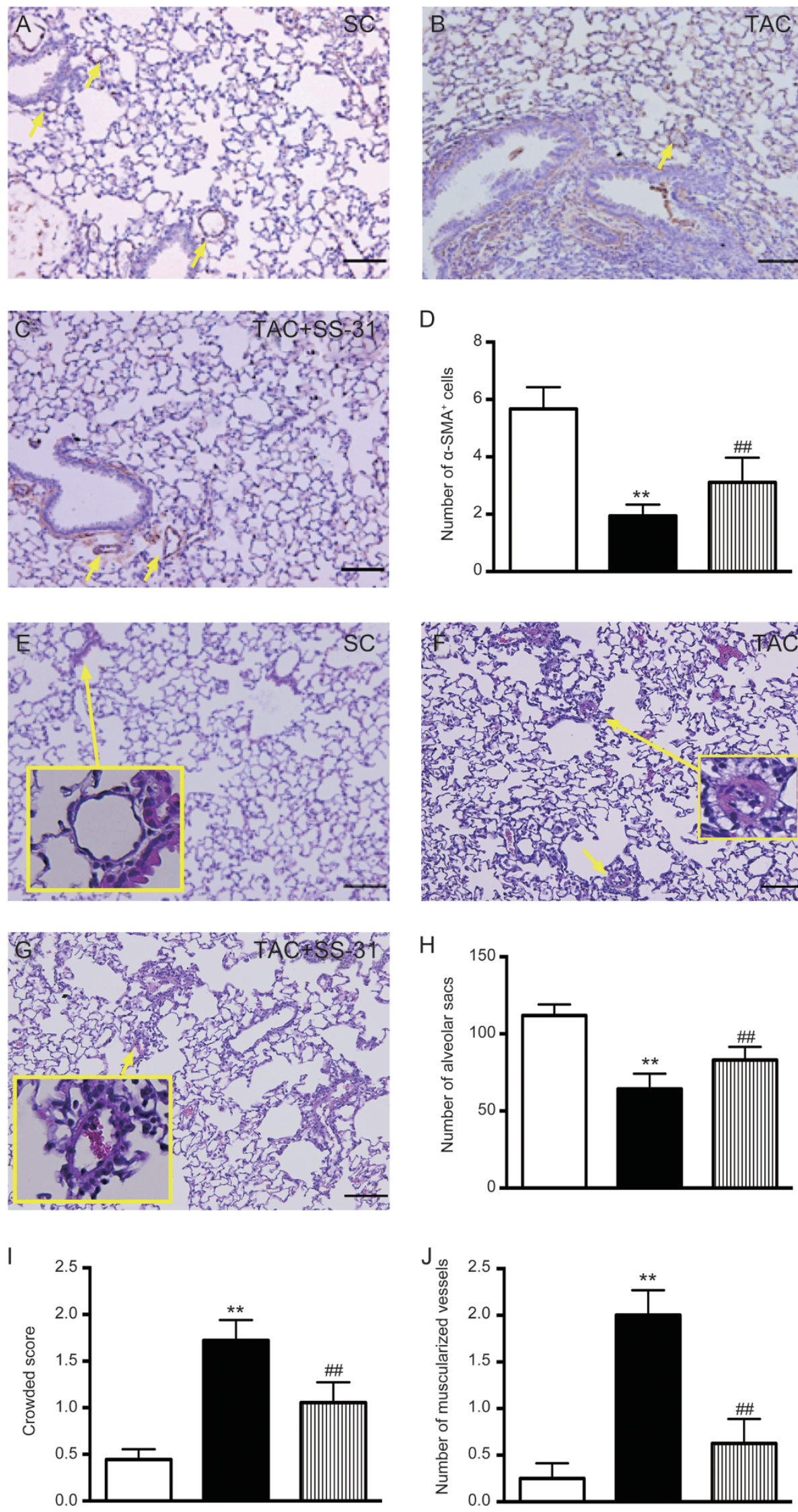

TAC

监血血 TAC+SS-31

D

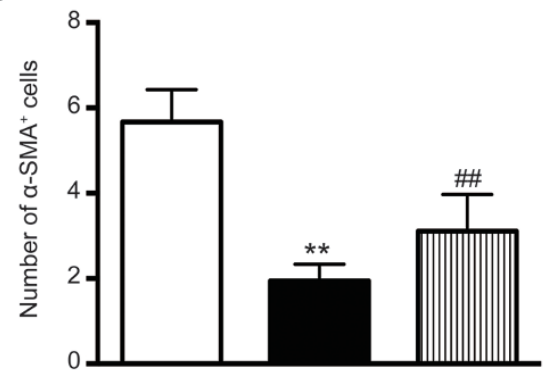

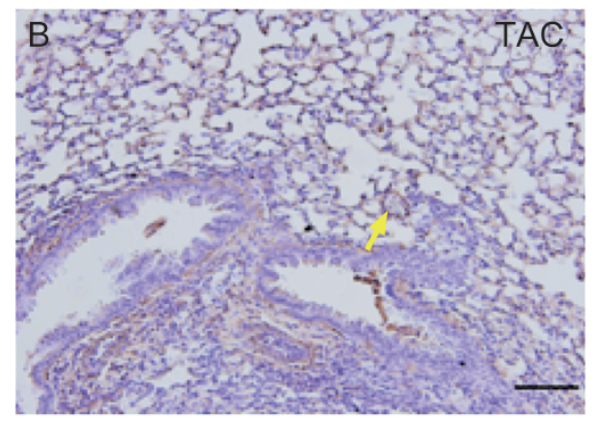

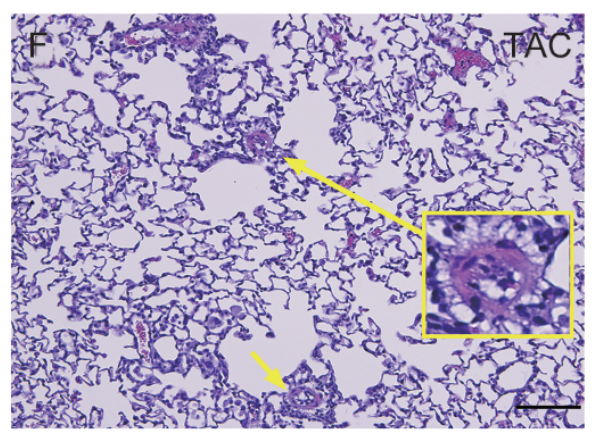

Figure 4. Histopathological findings in lung parenchyma at day 60 after the TAC procedure. (A-C) Microscopic findings of $\alpha$-smooth muscle actin staining in a lung section for the identification of the number of small vessels $\leq 15 \mu \mathrm{m}$ (yellow arrows) (×100). (D) Analytical results of the number of small vessels. The scale bars in the right lower corner represent $100 \mu \mathrm{m}$. (E-G) Microscopic findings of $H$ \& E staining in lung sections $(\times 100)$. The solidline square indicates the manifestation of the muscle layer of the pulmonary arteriole (PA). The appearance of muscularization of PA in TAC animals was remarkably more prominent than that in SC and TAC animals treated with SS-31. (H) Analytic results of the number of alveolar sacs. (I) Analytic results of crowded score. (J) Analytical results of the number of muscularized pulmonary arterioles. The scale bars in the right lower corner represent 100 $\mu \mathrm{m}$. All statistical analyses were performed by one-way ANOVA followed by Bonferroni multiple comparison post hoc test. $\mathrm{SC}=$ sham control. TAC=transverse aortic constriction. Mean \pm SEM. $n=8$ for each group. ${ }^{* *} P<0.01$ vs SC group. ${ }^{\#} P<0.01$ vs TAC group. 

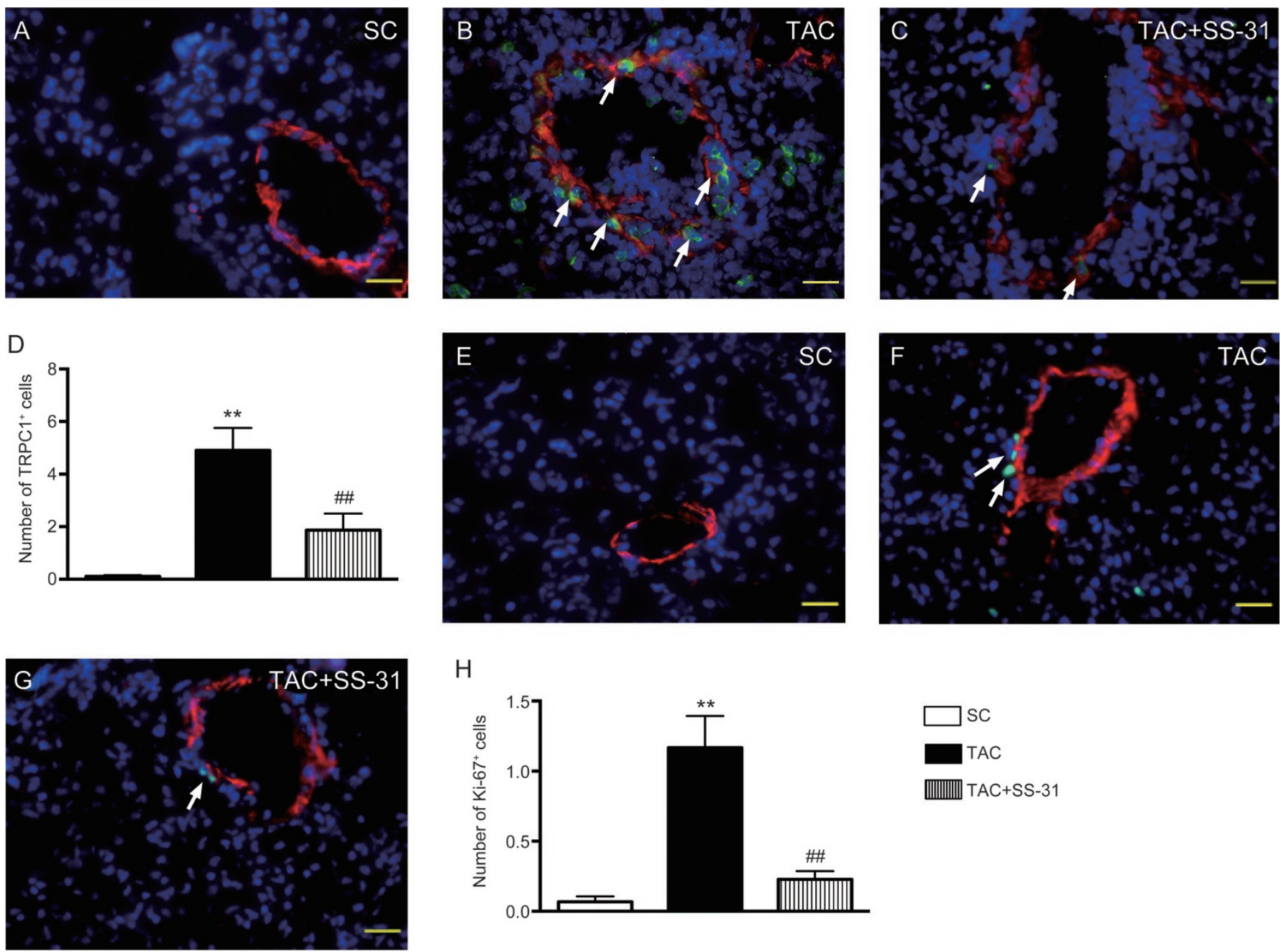

$\mathrm{H}$

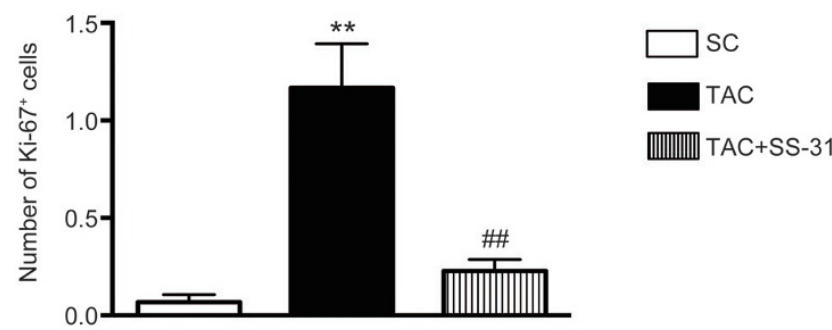

Figure 5. Immunofluorescent (IF) staining for identifying the expression levels of TRPC1 ${ }^{+}$and $\mathrm{Ki}-67^{+}$cells in pulmonary arteriolar smooth muscle at day 60 after the TAC procedure. (A-C) IF microscopic findings for the identification of positively stained transient receptor potential cation channel 1 (TRPC1) cells (white arrows) in the smooth muscle layer of the pulmonary arteriole (PA) ( $\times 400)$. (D) Analytic results of the number of TRPC $1^{+}$cells in the smooth muscle layer (PA). (E-G) IF microscopic findings for the identification of positively stained Ki-67 cells (white arrows) in the smooth muscle layer of the pulmonary arteriole $(\mathrm{PA})(\times 400)$. $(\mathrm{H})$ Analytical results of the number of $\mathrm{Ki}-67^{+}$cells in PA. The scale bars in the right lower corner represent $20 \mu \mathrm{m}$. All statistical analyses were performed by one-way ANOVA followed by Bonferroni multiple comparison post hoc test. $\mathrm{SC}=\mathrm{sham}$ control, TAC $=$ transverse aortic constriction. Mean \pm SEM. $n=8$ for each group. ${ }^{* *} P<0.01$ vs SC group. ${ }^{\# \#} P<0.01$ vs TAC group.

animals that received SS-31 treatment compared with SC animals (Figure 10).

\section{Discussion}

This study, which investigated the therapeutic impact of antioxidant peptide SS-31 on TAC-induced PAH in a murine model, yielded several striking findings. First, this study confirms that the TAC procedure was able to induce PAH that was remarkably suppressed by treatment with SS-31. Second, not only was the effectiveness of the treatment reflected in the improvement in hemodynamic status (ie, the reduction of RVSBP, an index of pulmonary arterial blood pressure) but it was also demonstrated in the anatomical and histopathological findings (ie, the reduction of total heart weight and the preservation of the architecture of the lung parenchyma and the right ventricle). The results of this study suggest that the observed therapeutic action mainly occurred through inhibition of the production of ROS, oxidative stress, inflammation, fibrosis, apoptosis, and mitochondrial damage.

SS-31 preserved the architectures of the lung parenchyma and the right ventricle and ameliorated TAC-induced PAH

Intriguingly, while several experimental studies have reported that mitochondrial-targeted compounds, including SS-31, protected the heart from ischemia-reperfusion injury and reactive hypertrophic cardiomyopathy ${ }^{[16,18-20]}$, there were no data to address whether mitochondrial-targeted peptide could also protect the right ventricle and lung parenchyma from passive $\mathrm{PAH}$-induced damage. The most important finding in the present study was the remarkable TAC-induced eleva- 

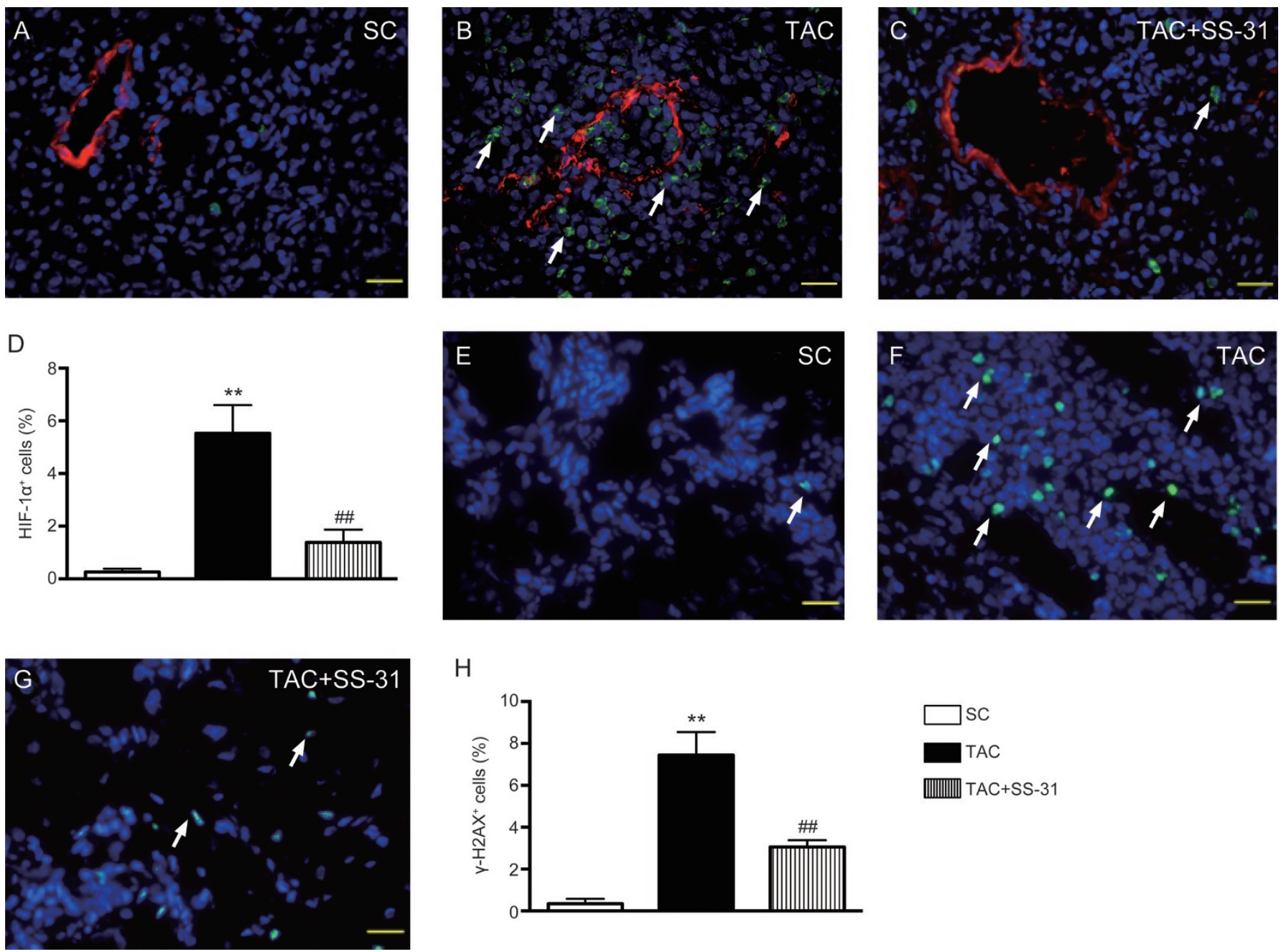

$\mathrm{H}$

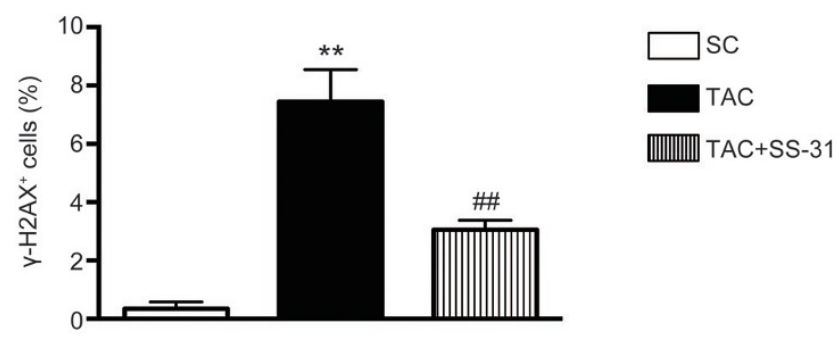

Figure 6. Immunofluorescent (IF) staining for identifying the expression levels of HIF-1 $\alpha^{+}$and $\mathrm{y}-\mathrm{H} 2 \mathrm{AX} \mathrm{X}^{+}$cells in lung parenchyma at day 60 after the TAC procedure. (A-C) IF microscopic findings for the identification of positively stained hypoxic inducible factor (HIF)-1 $\alpha$ cells (white arrows) in lung parenchyma ( $\times 400)$. (D) Analytic results of the number of HIF-1 $\alpha^{+}$cells. $(E-G)$ IF microscopic findings for the identification of positively stained $y-H 2 A X$ cells (white arrows) in lung parenchyma $(\times 400)$. (H) Analytic results of the number of $y-\mathrm{H}_{2} \mathrm{AX}^{+}$cells. The scale bars in the right lower corner represent $20 \mu \mathrm{m}$. All statistical analyses were performed by one-way ANOVA followed by Bonferroni multiple comparison post hoc test. SC $=$ sham control. TAC $=$ transverse aortic constriction. Mean \pm SEM. $n=8$ for each group. ${ }^{* *} P<0.01$ vs SC group. ${ }^{\# \#} P<0.01$ vs TAC group.

tion in RVSBP (ie, a reflection of pulmonary arterial systolic blood pressure) that was notably reduced after SS-31 treatment. Moreover, the microscopic architectural integrity of the lung parenchyma (ie, crowded score and the number of alveolar sacs) and the right ventricle (ie, fibrotic area and collagen deposition) was notably preserved in TAC animals after SS-31 treatment. Moreover, the results of analyses at the protein (ie, reduced BNP) and gene (ie, reduced expression levels of $\beta$-MHC, suppressed generation of ROS and inflammation biomarkers, and preserved a-MHC) levels also support the protective effects of SS-31 on RV in the present experimental setting of PAH. Importantly, in addition to extending the findings of previous studies that SS-31 protected the heart from ischemia-reperfusion injury and reactive hypertrophic cardiomyopathy ${ }^{[16,18-20]}$, the previously unreported results of the present study further highlight the possibility that SS-31 may be a useful accessory agent in the clinical treatment of patients with $\mathrm{PAH}$ in the near future, especially for patients with left-sided heart failure-induced PAH.

Mechanisms underlying alleviation of TAC-induced PAH and lung parenchymal damage after SS-31 treatment

An essential finding of the current study was the notable increase in the number of HIF-1 $\mathrm{a}^{+}$cells in lung parenchyma in TAC animals compared with those in sham-operated controls. Additionally, the numbers of $\mathrm{TRPC}^{+}$cells and $\mathrm{Ki}-67^{+}$ cells in PA were substantially higher in TAC animals than those in sham-operated controls. Furthermore, the protein expression levels of HIF- $\alpha$ and TRPCs (1, 2, 4 and 6) of lung tissues were also markedly upregulated in TAC animals compared with sham-operated controls. Interestingly, previous ${ }^{[26]}$ and recent ${ }^{[27]}$ studies have demonstrated that TRPC1 and 
A
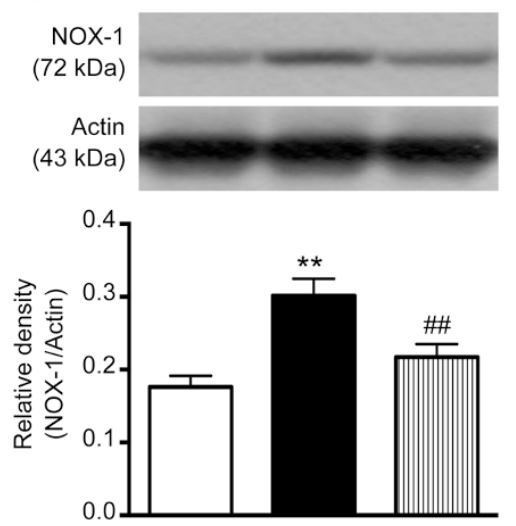

C
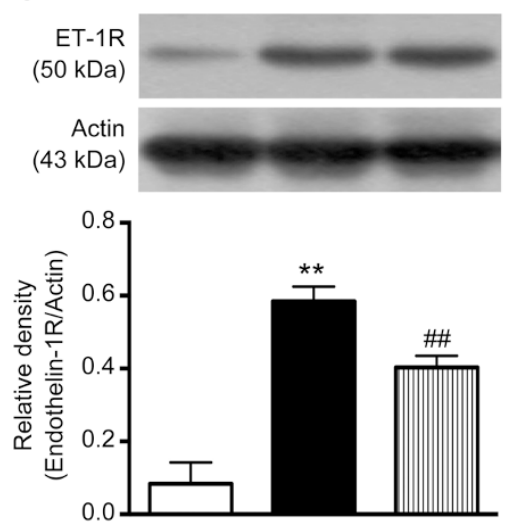

B
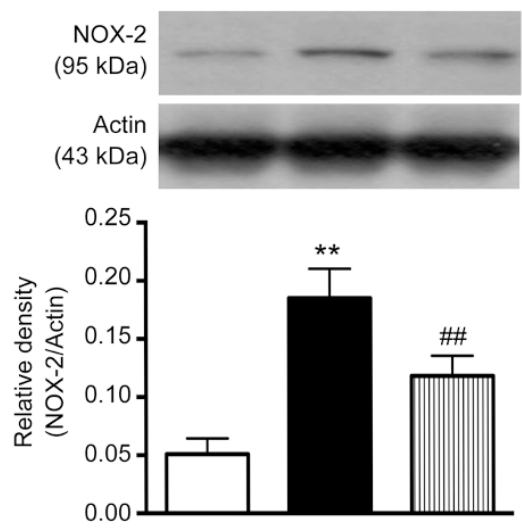

D

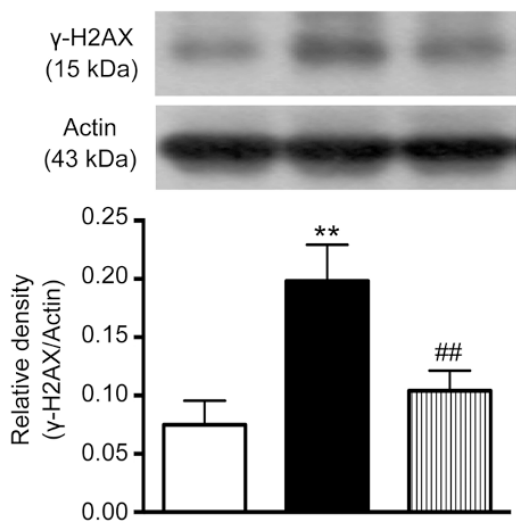

E
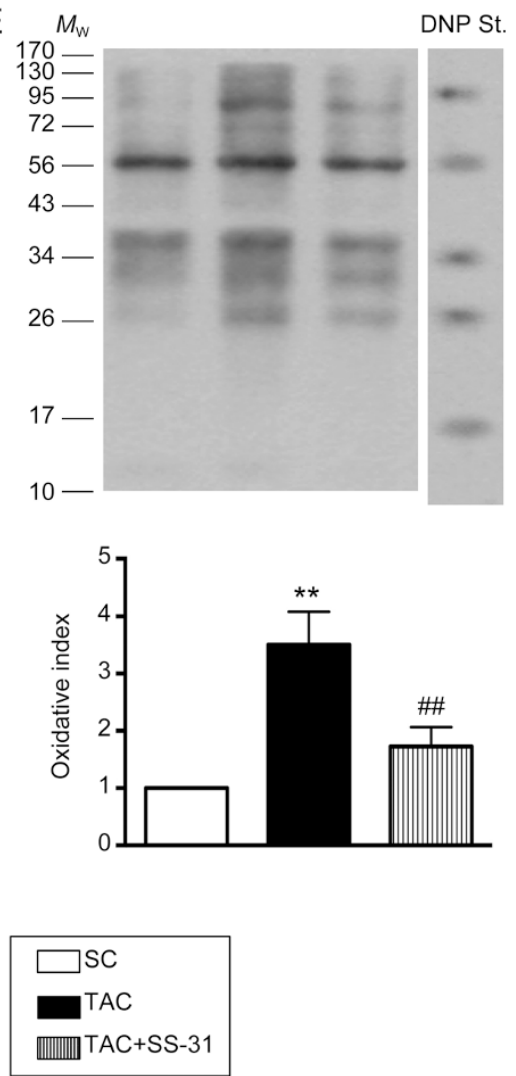

Figure 7. Protein expression levels of oxidative stress, endothelial and DNA damage markers in lung parenchyma at day 60 after the TAC procedure. (A) Protein expression of NOX-1. (B) Protein expression of NOX-2. (C) Protein expression of endothelin-1 receptor (ET-1R). (D) Protein expression of Y-H2AX. (E) Oxidized protein expression. $M_{\mathrm{W}}=$ molecular weight. $\mathrm{DNP}=1-3$ dinitrophenylhydrazone. All statistical analyses were performed by one-way ANOVA followed by Bonferroni multiple comparison post hoc test. $\mathrm{SC}=$ sham control. TAC=transverse aortic constriction. Mean $\pm S E M$. $n=8$ for each group. ${ }^{* *} P<0.01$ vs SC group. ${ }^{\# \#} P<0.01$ vs TAC group.

TRPC6 play a particularly crucial role in regulating smooth muscle cell contraction and proliferation in the murine model of hypoxia-induced PAH. Additionally, antigen Ki-67 is a nuclear protein that has been identified to be necessary for cellular proliferation ${ }^{[28]}$. Furthermore, the expression of HIF-a in PA has been shown to be strongly associated with hypoxicinduced $\mathrm{PAH}^{[27]}$. Accordingly, our findings, in addition to reinforcing the findings of those studies ${ }^{[26-28]}$, could, at least in part, explain the mechanisms involved in TAC-induced passive PAH. Importantly, these parameters were substantially attenuated in TAC animals after receiving SS-31 treatment.

Another essential finding of the present study was that the number of muscularized PA was notably increased, whereas the number of small vessels in lung parenchyma were notably reduced in TAC animals compared with those of sham-operated controls. Additionally, the protein expression of ET-R1 in the lung was significantly increased, whereas the protein expression of eNOS in the lung was notably reduced in TAC animals compared with those of sham-operated controls. Undoubtedly, an increase in the thickness of the PA smooth muscle layer and endothelin receptor 1 and a decrease in small vessel density and endothelial function in lung parenchyma are strongly associated with an increase in PASBP. Our findings once again explained why TAC induced PAH in the current study. Consistently, our previous studies have shown a significant increase in the number of muscularized PA and a notable decrease in the number of small vessels in lung parenchyma in monocrotaline-induced PAH in rats ${ }^{[21,27,29]}$. In this way, our findings not only corroborate those of the previous studies ${ }^{[21,27,29]}$ but also illustrate the positive therapeutic impact of SS-31 and the possible mechanisms by which it acts in the present experimental setting.

A principal finding of the current study is the significantly augmented DNA damage (ie, $\gamma-\mathrm{H}_{2} \mathrm{AX}^{+}$cells); the increase in protein expression levels of fibrotic (Smad3 and TGF- $\beta$ ), apoptotic (mitochondrial Bax, cleaved caspase 3 and PARP) and energy exhausted (cytosolic cytochrome $c$ ) biomarkers, and the remarkable reduction in the expression levels of anti-fibrotic (Smad1/5 and BMP-2) and energy preservative (mitochondrial cytochrome $c$ ) biomarkers in TAC animals compared to those in sham-operated controls. In concert with the results of the present investigation, our previous studies ${ }^{[21,27,29]}$ have also 
A
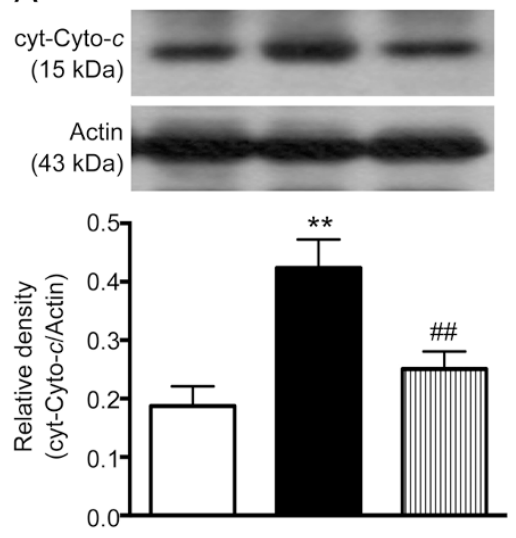

D
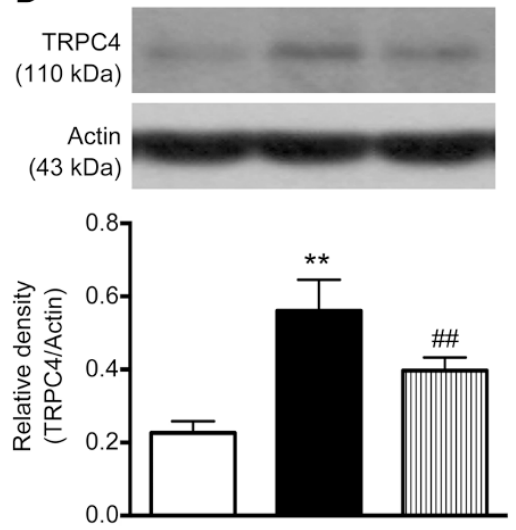

B
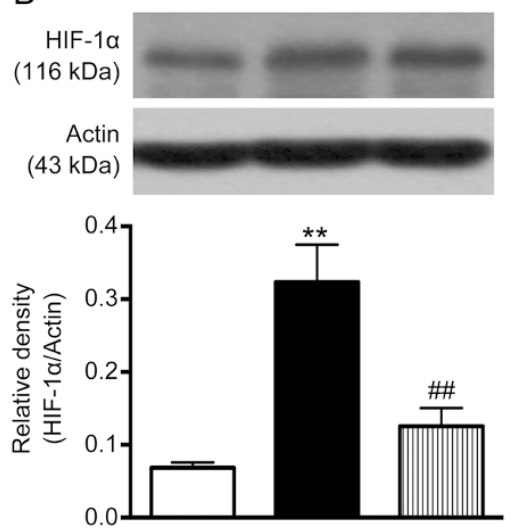

E
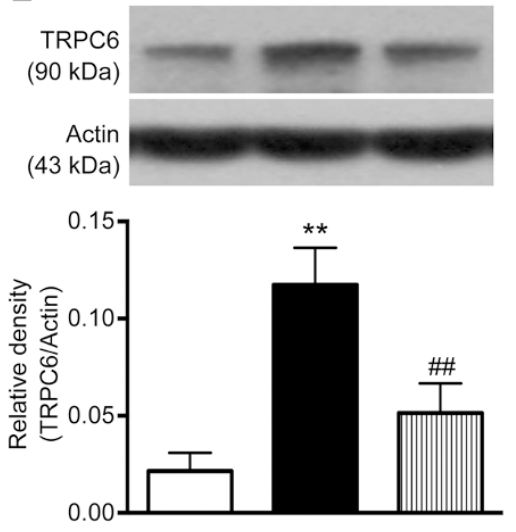

SC
C
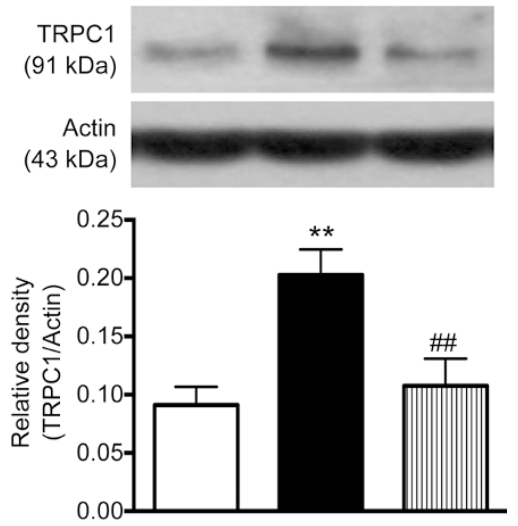

$\mathrm{F}$
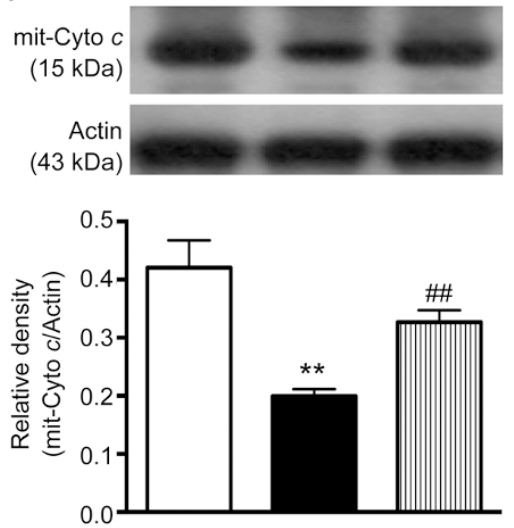

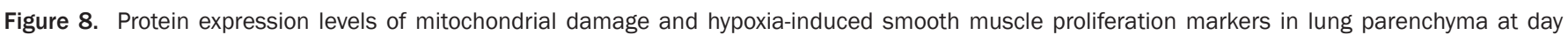

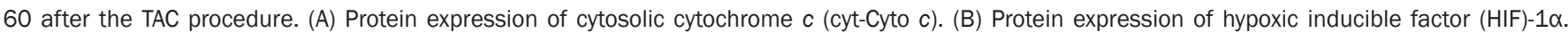

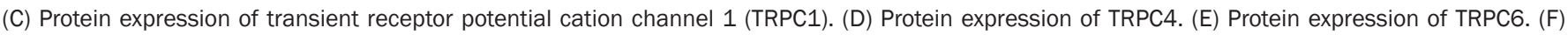

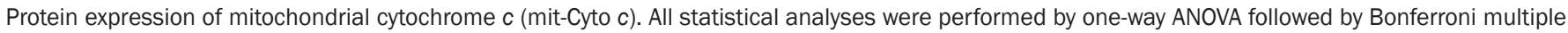

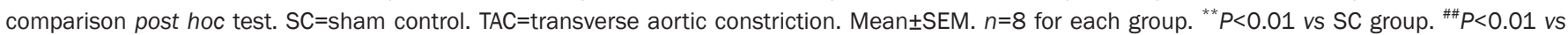
TAC group.

revealed marked elevations of these biomarkers in animals with monocrotaline-induced PAH. The substantial reversal of expression levels of these parameters after SS-31 treatment further suggests the suppression of these pathological processes in reinforcing the observed therapeutic effects in a murine model of PAH.

Another principal finding of the present study is the significantly progressively increased antioxidants (HO-1, NQO-1, GR and GPx) from sham-operated controls to TAC animals with SS-31 treatment. We propose that the observation that these parameters were significantly higher in TAC animals than in sham-operated controls could be an effect of the cells response to stress stimulation. Intriguingly, a previous study has also found that antioxidant biomarkers were markedly increased in lung parenchyma in response to acute ischemiareperfusion injury ${ }^{[24]}$. In this way, our finding was consistent with the finding of the previous study ${ }^{[24]}$. Additionally, SS-31 has also been found to have antioxidant abilities alongside its intrinsic mitochondrial protective capacities ${ }^{[17,18,20]}$. Therefore, our finding of a further significant increase in the antioxidants in TAC animals after receiving SS-31 treatment, in addition to strengthening the finding of the previous study ${ }^{[24]}$, could explain why cell death was remarkably reduced in animals after receiving SS-31 treatment.

\section{Study limitations}

This study has some limitations. First, because this study was designed to create only a moderate LV pressure overload to maximize animal survival for the purpose of following the progression of hypertrophic cardiomyopathy, the effect of SS-31 on animal survival under acute severe LV pressure overload was not investigated. Second, information regarding the long-term (ie, over 3 months) therapeutic effect of SS-31 against hypertrophic cardiomyopathy and on survival was 
A
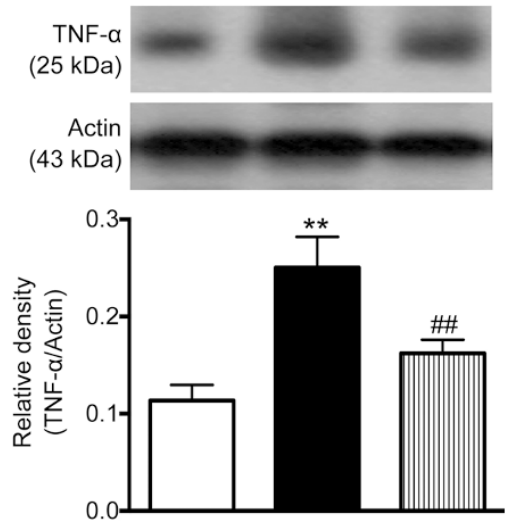

D
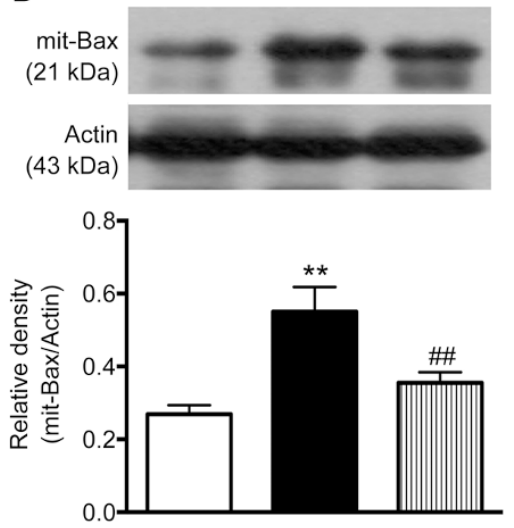

G
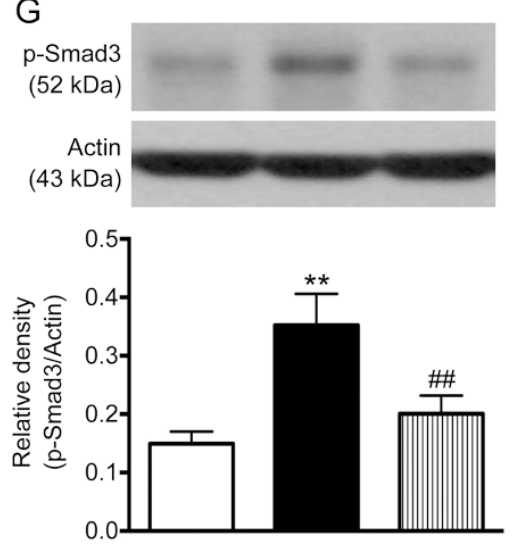

B
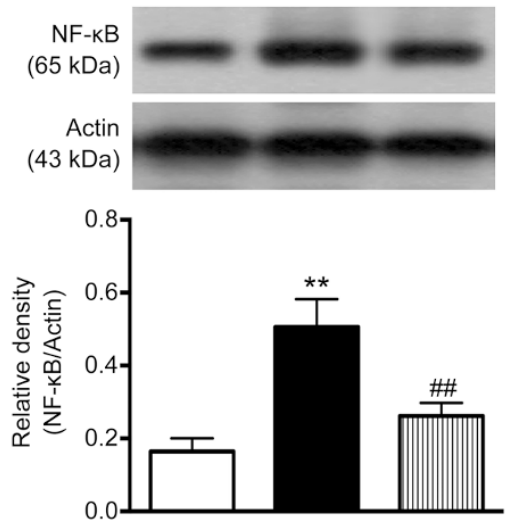

E
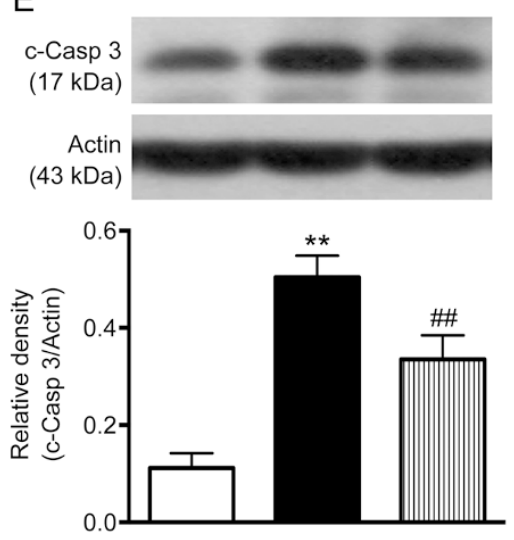

$\mathrm{H}$
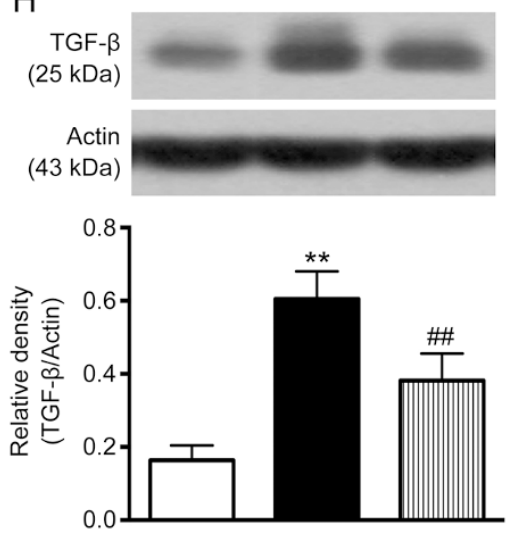

C
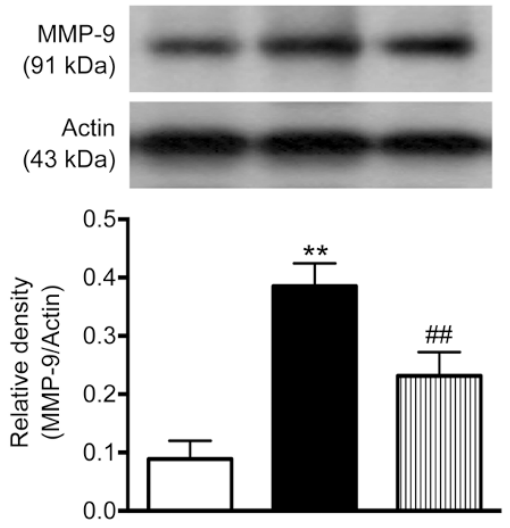

F
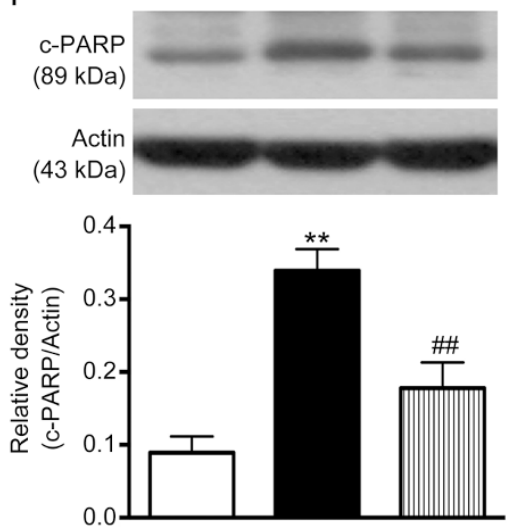

Figure 9. Protein expression levels of inflammatory, apoptotic and fibrotic markers in lung parenchyma at day 60 after the TAC procedure. (A) Protein expression of tumor necrosis factor (TNF)- $\alpha$. (B) Protein expression of nuclear factor (NF)-KB. (C) Protein expression of matrix metalloproteinase (MMP)9. (D) Protein expression of mitochondrial Bax (mit-Bax). (E) Protein expression of cleaved caspase (c-Casp) 3. (F) Protein expression of cleaved poly (ADP-ribose) polymerase (c-PARP). (G) Protein expression of phosphorylated (p)-Smad3. (H) Protein expression of transforming growth factor (TGF)- $\beta$. All statistical analyses were performed by one-way ANOVA followed by Bonferroni multiple comparison post hoc test. SC=sham control. TAC=Transverse aortic constriction. Mean \pm SEM. $n=8$ for each group. ${ }^{* *} P<0.01$ vs SC group. ${ }^{\# \#} P<0.01$ vs TAC group.

not provided by the present study. Third, because left-sided heart failure-induced PAH caused lung parenchymal injuries through multiple signaling pathways, the results of the present study could not identify the most important pathway.

In conclusion, the results of this study show that the antioxi- dant peptide SS-31 markedly attenuated TAC-induced PAH as well as lung parenchymal and RV damage. These findings may warrant further investigation into the possible clinical application of this agent, particularly in patients who are refractory to current treatment strategies. 
A
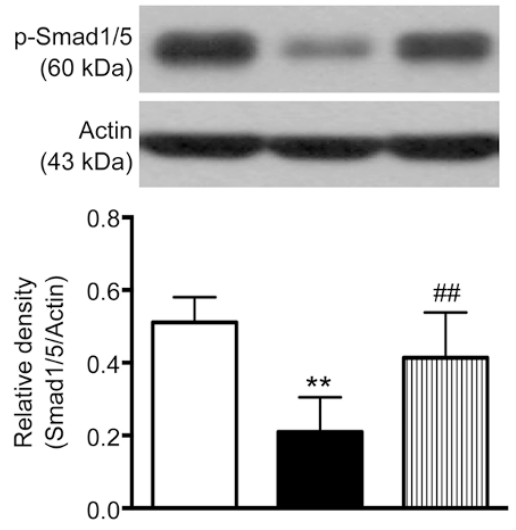

D
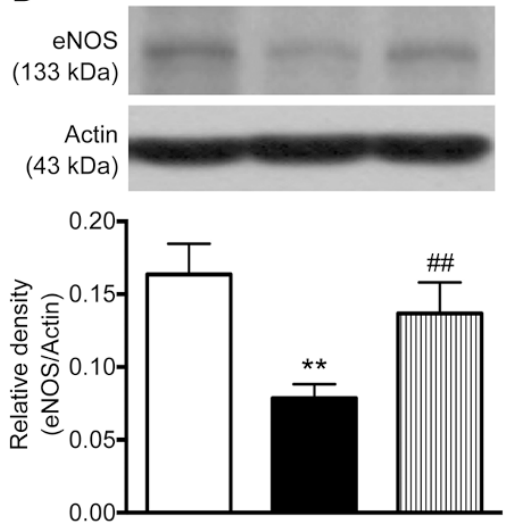

G
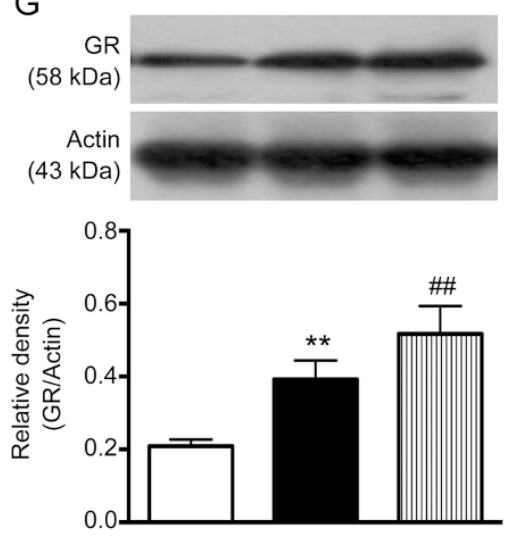

B
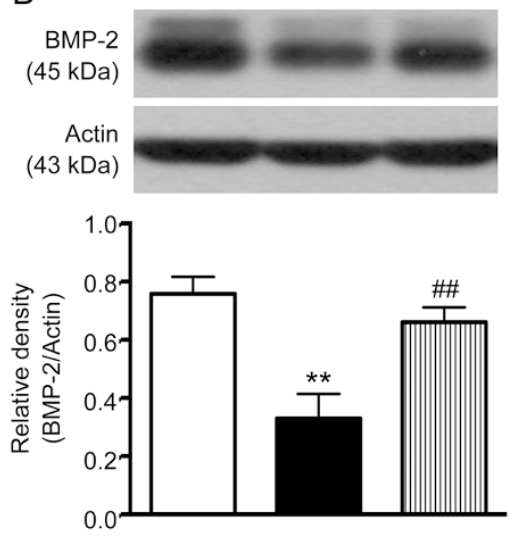

E
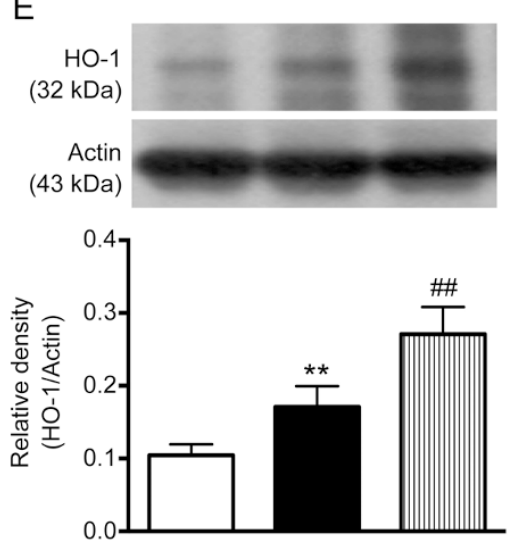

$\mathrm{H}$
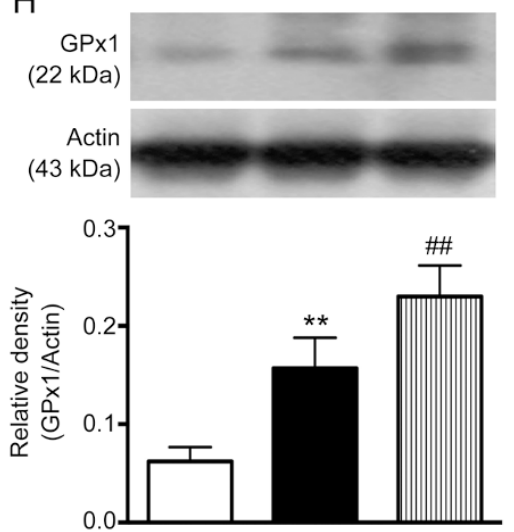

C
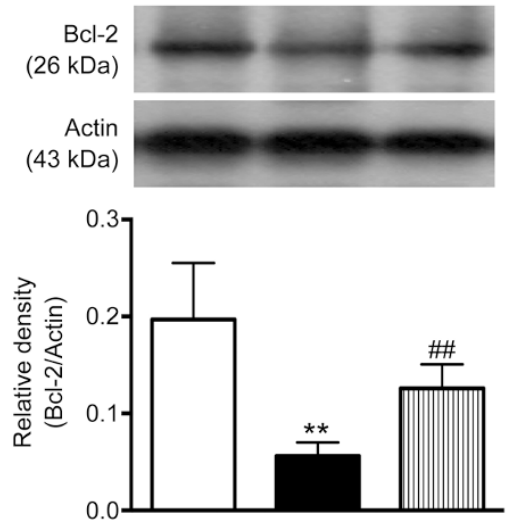

F
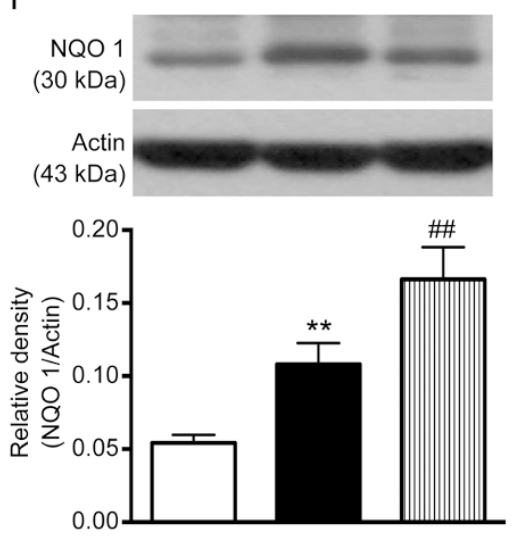

Figure 10. Protein expression levels of anti-fibrotic, anti-inflammatory, antioxidant and endothelial function biomarkers in lung parenchyma at day 60 after the TAC procedure. (A) Protein expression of p-Smad1/5. (B) Protein expression of bone morphogenetic protein (BMP)-2. (C) Protein expression of $\mathrm{Bcl}-2$. (D) Protein expression of endothelial nitric oxide synthase (eNOS). (E) Protein expression of heme oxygenase (HO)-1. (F) Protein expression of $\mathrm{NAD}(\mathrm{P}) \mathrm{H}$ quinone oxidoreductase (NQO) 1. (G) Protein expression of glutathione reductase (GR). (H) Protein expression of glutathione peroxidase (GPx). All statistical analyses were performed by one-way ANOVA followed by Bonferroni multiple comparison post hoc test. $\mathrm{SC}=\mathrm{Sham}$ control. TAC=Transverse aortic constriction. Mean \pm SEM. $n=8$ for each group. ${ }^{* *} P<0.01$ vs SC group. ${ }^{\# \#} P<0.01$ vs TAC group.

\section{Acknowledgements}

This study was supported by a program grant from Chang Gung Memorial Hospital, Chang Gung University (No CMRPG8C0131).

\section{Author contribution}

Hung-I LU, Tien-hung HUANG and Hon-kan YIP designed the study; Pei-hsun SUNG, Yung-lung CHEN and Yi-ling 
CHEN performed the experiments; Sarah CHUA, Han-tan CHAI, Sheng-ying CHUNG and Chu-feng LIU contributed new reagents or analytic tools; Cheuk-kwan SUN, Hsueh-wen CHANG and Yen-yi ZHEN analyzed data; Cheuk-kwan SUN, Fan-yen LEE and Hon-kan YIP wrote the paper. An author may list more than one contribution, and more than one author may have contributed to the same aspect of the study.

\section{References}

1 Binanay C, Califf RM, Hasselblad V, O'Connor CM, Shah MR, Sopko G, et al. Evaluation study of congestive heart failure and pulmonary artery catheterization effectiveness: the ESCAPE trial. JAMA 2005; 294 : 1625-33.

2 Chang PP, Chambless LE, Shahar E, Bertoni AG, Russell SD, Ni H, et al. Incidence and survival of hospitalized acute decompensated heart failure in four US communities (from the Atherosclerosis Risk in Communities Study). Am J Cardiol 2014; 113: 504-10.

3 Sotomi Y, Sato N, Kajimoto K, Sakata Y, Mizuno M, Minami Y, et al. Impact of pulmonary artery catheter on outcome in patients with acute heart failure syndromes with hypotension or receiving inotropes: from the ATTEND Registry. Int J Cardiol 2014; 172: 165-72.

4 Thenappan T, Shah SJ, Gomberg-Maitland M, Collander B, Vallakati A, Shroff $P$, et al. Clinical characteristics of pulmonary hypertension in patients with heart failure and preserved ejection fraction. Circ Heart Fail 2011; 4: 257-65.

5 Vachiery JL, Adir Y, Barbera JA, Champion H, Coghlan JG, Cottin V, et al. Pulmonary hypertension due to left heart diseases. J Am Coll Cardiol 2013; 62: D100-8.

6 Cui Y, Doupe M, Katz A, Nyhof P, Forget EL. Economic evaluation of Manitoba Health Lines in the management of congestive heart failure. Healthc Policy 2013; 9: 36-50.

7 Stewart S, Carrington MJ, Marwick TH, Davidson PM, Macdonald P, Horowitz JD, et al. Impact of home versus clinic-based management of chronic heart failure: the WHICH? (Which Heart Failure Intervention Is Most Cost-Effective \& Consumer Friendly in Reducing Hospital Care) multicenter, randomized trial. J Am Coll Cardiol 2012; 60: 1239-48.

8 Gasparini M, Leclercq C, Yu CM, Auricchio A, Steinberg JS, Lamp B, et al. Absolute survival after cardiac resynchronization therapy according to baseline QRS duration: a multinational 10-year experience: data from the Multicenter International CRT Study. Am Heart J 2014; 167 : 203-9 e1.

9 Granger CB, McMurray JJ, Yusuf S, Held P, Michelson EL, Olofsson B, et al. Effects of candesartan in patients with chronic heart failure and reduced left-ventricular systolic function intolerant to angiotensinconverting-enzyme inhibitors: the CHARM-Alternative trial. Lancet 2003; 362: 772-6.

10 Meune C, Wahbi K, Duboc D, Weber S. Meta-analysis of renin-angiotensin-aldosterone blockade for heart failure in presence of preserved left ventricular function. J Cardiovasc Pharmacol Ther 2011; 16 : 368-75.

11 Tsutsui H, Kinugawa S, Matsushima S. Oxidative stress and heart failure. Am J Physiol Heart Circ Physiol 2011; 301: H2181-90.

12 Whiteman M, Spencer JP, Szeto HH, Armstrong JS. Do mitochondriotropic antioxidants prevent chlorinative stress-induced mitochondrial and cellular injury? Antioxid Redox Signal 2008; 10: 641-50.

13 Zhao K, Luo G, Giannelli S, Szeto HH. Mitochondria-targeted peptide prevents mitochondrial depolarization and apoptosis induced by tert- butyl hydroperoxide in neuronal cell lines. Biochem Pharmacol 2005; 70: 1796-806.

14 Sharma R, Davidoff MN. Oxidative stress and endothelial dysfunction in heart failure. Congest Heart Fail 2002; 8: 165-72.

15 Tsutsui H, Kinugawa S, Matsushima S. Mitochondrial oxidative stress and dysfunction in myocardial remodelling. Cardiovasc Res 2009; 81: 449-56.

16 Zhao K, Zhao GM, Wu D, Soong Y, Birk AV, Schiller PW, et al. Cellpermeable peptide antioxidants targeted to inner mitochondrial membrane inhibit mitochondrial swelling, oxidative cell death, and reperfusion injury. J Biol Chem 2004; 279: 34682-90.

17 Birk AV, Liu S, Soong Y, Mills W, Singh P, Warren JD, et al. The mitochondrial-targeted compound SS-31 re-energizes ischemic mitochondria by interacting with cardiolipin. J Am Soc Nephrol 2013; 24: 1250-61.

18 Dai DF, Chen T, Szeto H, Nieves-Cintron M, Kutyavin V, Santana LF, et al. Mitochondrial targeted antioxidant peptide ameliorates hypertensive cardiomyopathy. J Am Coll Cardiol 2011; 58: 73-82.

19 Kloner RA, Hale SL, Dai W, Gorman RC, Shuto T, Koomalsingh KJ, et al. Reduction of ischemia/reperfusion injury with bendavia, a mitochondria-targeting cytoprotective peptide. J Am Heart Assoc 2012; 1: e001644.

20 Szeto HH. Mitochondria-targeted cytoprotective peptides for ischemiareperfusion injury. Antioxid Redox Signal 2008; 10: 601-19.

21 Sun CK, Lin YC, Yuen CM, Chua S, Chang LT, Sheu JJ, et al. Enhanced protection against pulmonary hypertension with sildenafil and endothelial progenitor cell in rats. Int J Cardiol 2012; 162: 45-58.

22 Chen YT, Tsai TH, Yang CC, Sun CK, Chang LT, Chen HH, et al. Exendin-4 and sitagliptin protect kidney from ischemia-reperfusion injury through suppressing oxidative stress and inflammatory reaction. J TransI Med 2013; 11: 270.

23 Chen YT, Yang CC, Zhen YY, Wallace CG, Yang JL, Sun CK, et al. Cyclosporine-assisted adipose-derived mesenchymal stem cell therapy to mitigate acute kidney ischemia-reperfusion injury. Stem Cell Res Ther 2013; 4: 62.

24 Yip HK, Chang YC, Wallace CG, Chang LT, Tsai TH, Chen YL, et al. Melatonin treatment improves adipose-derived mesenchymal stem cell therapy for acute lung ischemia-reperfusion injury. J Pineal Res 2013; 54: 207-21.

25 Chua S, Lee FY, Tsai TH, Sheu JJ, Leu S, Sun CK, et al. Inhibition of dipeptidyl peptidase-IV enzyme activity protects against myocardial ischemia-reperfusion injury in rats. J TransI Med 2014; 12: 357.

26 Lin MJ, Leung GP, Zhang WM, Yang XR, Yip KP, Tse CM, et al. Chronic hypoxia-induced upregulation of store-operated and receptor-operated $\mathrm{Ca}^{2+}$ channels in pulmonary arterial smooth muscle cells: a novel mechanism of hypoxic pulmonary hypertension. Circ Res 2004; 95: 496-505.

27 Sun CK, Zhen YY, Lu HI, Sung PH, Chang LT, Tsai TH, et al. Reducing TRPC1 expression through liposome-mediated siRNA delivery markedly attenuates hypoxia-induced pulmonary arterial hypertension in a murine model. Stem Cells Int 2014; 2014: 316214.

28 Scholzen T, Gerdes J. The Ki-67 protein: from the known and the unknown. J Cell Physiol 2000; 182: 311-22.

29 Yen CH, Leu S, Lin YC, Kao YH, Chang LT, Chua S, et al. Sildenafil limits monocrotaline-induced pulmonary hypertension in rats through suppression of pulmonary vascular remodeling. J Cardiovasc Pharmacol 2010; 55: 574-84. 